\title{
ARBITRARILY SHAPED VIRTUAL-OBJECT VIDEO COMPRESSION
}

\author{
A Thesis \\ Presented in Partial Fulfillment of the Requirements for \\ the Degree Master of Science in the \\ Graduate School of The Ohio State University \\ By
}

Naresh Sharma, B.E.

$* * * * *$

The Ohio State University

2009

Master's Examination Committee:

Approved by

Professor Yuan F. Zheng, Adviser

Professor Ashok Krishnamurthy

Adviser

Graduate Program in Electrical and Computer Engineering 
(c) Copyright by

Naresh Sharma

2009 


\begin{abstract}
With the advancements in multimedia technologies, effective video compression has become more and more important. The size of the video files is always increasing with the increasing camera resolutions and because of never ending demand for better quality video signals. On the other hand, there is a limit to the available storage space and transmission bandwidth. Therefore, it is important to have good quality videos at low bit-rates. However, the widely established compression standards suffer from the annoying blocking artifacts at very low bit-rates and therefore, are not suitable for video coding at low bit-rates. The reason for the blocking artifacts is the usage of block based discrete cosine transform in these established compression methods. One good solution to this problem is to use wavelet transform which can be directly applied to the whole image at once, and thus, do not suffer from blocking artifacts. In addition, object based compression is also gaining in popularity because of the flexibility it provides to the end-user, and because of its widely believed potential to deliver good quality videos at very low bit-rates.

Therefore, this dissertation develops a compression method that further explores the advantages of object based compression, and uses shape adaptive wavelet transform for the coding of the arbitrarily shaped virtual-object thereby avoiding any blocking artifacts.
\end{abstract}


An arbitrarily shaped virtual-object compression method is developed. Method extracts the changing portion of the video as a 3D arbitrarily shaped virtual object from the non-changing portion termed as background. Arbitrarily shaped virtual object is coded using 3D wavelet compression whereas stationary background is coded using 2D wavelet compression. Experimental results demonstrate that the newly developed method outperforms 3D wavelet compression and the rectangular virtualobject compression by achieving higher compression ratio at a higher PSNR. 
This is dedicated to my parents 


\section{ACKNOWLEDGMENTS}

First of all, I would like to express my sincere gratitude to my advisor Professor Yuan F. Zheng. Without his guidance, encouragement, and financial support throughout my years in Masters study at The Ohio State University, this dissertation would not have been completed. He gave me a direction in my career and a quality research work in the field of video compression during my time as his student. He taught me how to approach the research related problems, and I always learned from his innovative solutions to many research problems. He always inspired me to work hard and has instilled the belief in me that with hard work, any goal can be achieved. It has truly been a privilege to work under his guidance.

A special thanks to Professor Ashok K. Krishnamurthy for his time in serving on my committee. His technical feedback has been really helpful in completing this dissertation in a better manner.

I also show my appreciation to my colleges in The Multimedia and Robotics Laboratory at The Ohio State University. Special thanks to Mr. Junda Zhu, Mr. Yuanwei Lao, and Mr. Rittavee Matungka to help with many technical problems from time to time. I would also like to thank Mr. Kevin Huggins, Mr. Liang Yuan, and Ms. Yen-Lun Chen for their continual help and warmth friendship. It has been a wonderful journey because of them. 
I dedicate this disseration to my parents Mr. Om Prakash Sharma and Mrs. Soma Devi, who never doubted my dreams and have always been there for me. Without their constant encouragement and undying love, it would not have been possible for me to achieve my goals. 


\section{VITA}

August 19, $1982 \ldots \ldots \ldots \ldots \ldots \ldots$. . . B . Born - Hamirpur, India

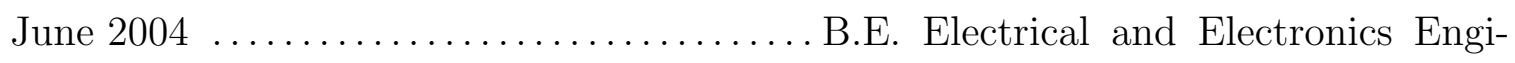
neering, Birla Institute of Technology and Science, Pilani, India

July 2004 - August 2007 ............... Software Engineer, STMicroelectronics Pvt. Ltd., Greater Noida, India

September $2007 \ldots \ldots \ldots \ldots \ldots \ldots \ldots$. Joined The Ohio State University for M.S. in Electrical and Computer Engineering

January 2008 - Present ............... Graduate Research Associate, Electrical and Computer Engineering, The Ohio State University, Columbus, $\mathrm{OH}$

\section{FIELDS OF STUDY}

Major Field: Computer Engineering

Studies in:

Video Compression

Signal Processing

Computer Architecture 


\section{TABLE OF CONTENTS}

Page

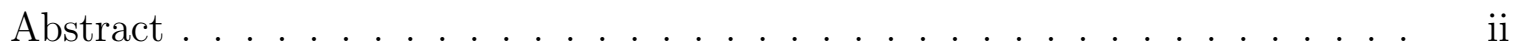

Dedication ............................ iv

Acknowledgments . . . . . . . . . . . . . . . . . . v

Vita ........................... vii

List of Tables . . . . . . . . . . . . . . . . .

List of Figures $\ldots \ldots \ldots \ldots \ldots \ldots$ xi

Chapters:

1. Introduction . . . . . . . . . . . . . . . . . . 1

1.1 Video Compression Standards . . . . . . . . . . . . . . . . . 2

1.2 Motivations . . . . . . . . . . . . . . . . . 6

1.2.1 Wavelet Compression vs Discrete Cosine Transform Compression . . . . . . . . . . . . . 6

1.2.2 Arbitrarily Shaped Virtual-Object Compression . . . . . . 9

1.3 Dissertation Overview . . . . . . . . . . . . . . . . . . 10

2. Shape Adaptive Wavelet Transform . . . . . . . . . . . . . . . . . . . 12

2.1 Shape Adaptive Wavelet Transform . . . . . . . . . . . . . . . . 12

2.1.1 Boundary Extension . . . . . . . . . . . . . . . . . . 13

2.1.2 Arbitrary Length Wavelet Transform . . . . . . . . . . 14

2.1.3 Subsampling Strategy . . . . . . . . . . . . . . . . 15

2.2 2-D Shape Adaptive Wavelet Transform . . . . . . . . . . . 16

2.3 Summary . . . . . . . . . . . . . . . . . . . . . . 18 
3. Arbitrarily Shaped Virtual-Object Video Compression _. . . . . . . . 19

3.1 Introduction . . . . . . . . . . . . . . . . . . . . . . 19

3.2 Virtual-Object Video Compression . . . . . . . . . . . . . 22

3.2 .1 Virtual Object . . . . . . . . . . . . . . . . . 23

3.2 .2 Virtual-Object Extraction . . . . . . . . . . . . . . . 24

3.2 .3 Virtual-Object Video Compression . . . . . . . . . . . 26

3.2.4 Virtual-Object Video Compression Results . . . . . . . . . . 26

3.3 Arbitrarily Shaped Virtual Object Compression . . . . . . . . . 27

3.3.1 Arbitrarily Shaped Virtual-Object . . . . . . . . . . . . 29

3.3.2 Arbitrarily Shaped Virtual-Object Video Compression . . . 32

3.3 .3 Number of Virtual Objects . . . . . . . . . . . . . . . 33

3.3 .4 Group of Frames . . . . . . . . . . . . . . . . . . . . . 38

3.4 Conclusions . . . . . . . . . . . . . . . . . . . 40

4. Experiments and Results . . . . . . . . . . . . . . . . . . . . 41

4.1 Performance Comparison between 3D Wavelet and Arbitrarily Shaped Virtual-Object Compression . . . . . . . . . . . . . . . . . . . 41

4.1.1 Single Arbitrarily Shaped Virtual Object . . . . . . . . . . . 41

4.1.2 Multiple Arbitrarily Shaped Virtual Object . . . . . . . . . 42

4.2 Performance Comparison between Rectangular Virtual-Object and Arbitrarily Shaped Virtual-Object Compression . . . . . . . . . . 44

4.2.1 Single Arbitrarily Shaped Virtual Object . . . . . . . . . . 46

4.2.2 Multiple Arbitrarily Shaped Virtual Object . . . . . . . . . 46

4.2.3 Video Sequence with Multiple Moving Objects . . . . . . 50

4.3 Conclusions . . . . . . . . . . . . . . . . . . . . . . 51

5. Contributions and Future Work . . . . . . . . . . . . . . . 55

5.1 Contributions . . . . . . . . . . . . . . . 55

5.2 Future Works . . . . . . . . . . . . . . . . . . . 56

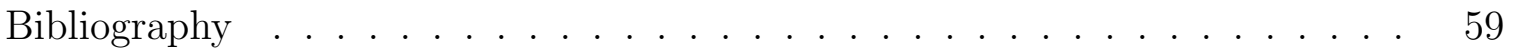




\section{LIST OF TABLES}

Table

Page

4.1 Summary of Compression results . . . . . . . . . . . . . . 50 


\section{LIST OF FIGURES}

Figure

Page

1.1 A general MPEG video encoder ... . . . . . . . . . . . . 4

2.1 Symmetric extension for an odd symmetric biorthogonal wavelet filter at the leasing and trailing boundaries . . . . . . . . . . . . . . . . . 13

3.1 Virtual-Object Definitions . . . . . . . . . . . . . . . 24

3.2 Virtual-Object Video Compression . . . . . . . . . . . . . . 27

3.3 Comparison between 3-D compression and Virtual-Object Compresson using "10TV" sequence (frame \#22 is shown), (a) 3D wavelet compression. Compression ratio $=36: 1$, Average PSNR $=31.12$, (b) VirtualObject compression. Compression Ratio $=40: 1$, Average PSNR $=$

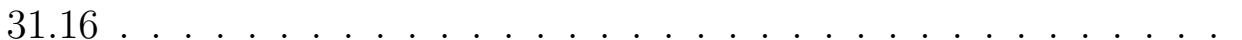

3.4 Arbitrarily Shaped Virtual-Object Extraction(frame \#22 of "10TV" image sequence is shown). (a) Original Frame, (b) Rectangular VirtualObject, and (c) Arbitrarily Shaped Virtual-Object. Note that the stationary background is intentionally shown white for clarity purpose. Only the colored portion shown in (b) and (c) belongs to the virtualobject and is compressed in 3D. As colored portion is much less in (c), compression ratio will be higher in arbitrarily shaped virtual-object compression method .................... 32

3.5 Arbitrarily Shaped Virtual-Object Video Compression . . . . . . . . . 34

3.6 Multiple Arbitrarily Shaped Virtual-Objects Video Compression . . . 36 
4.1 Comparison between 3-D compression and Single Arbitrarily Shaped Virtual-Object Compression using "10TV" sequence (frame \#22 is shown), (a) 3D wavelet compression. Compression ratio $=23: 1$, Average PSNR $=31.53$, (b) Extracted Arbitrarily Shaped Virtual Object, the whole colored region is considered to be belonging to the same single virtual object and is bounded by single rectangular box of dimensions same as the frame size ( background intentionally shown white for clarity) (c) Single Arbitrarily Shaped Virtual-Object Compression. Compression Ratio $=42.22: 1$, Average PSNR $=33.83 \ldots . .$.

4.2 Comparison between 3-D compression and Multiple Arbitrarily Shaped Virtual-Object Compression using "10TV" sequence (frame \#22 is shown), (a) 3D wavelet compression. Compression ratio $=23: 1$, Average PSNR $=31.53$, (b) Extracted Multiple Arbitrarily Shaped Virtual Objects along with their bounding boxes ( background intentionally shown white for clarity) (c) Multiple Arbitrarily Shaped Virtual-Object Compression. Compression Ratio $=47: 1$, Average PSNR $=33.76$.

4.3 PSNR comparison between 3D wavelet, single arbitrarily shaped virtualobject (SASVO), and multiple arbitrarily shaped virtual-object (MASVO) compression methods . . . . . . . . . . . . . .

4.4 Comparison between virtual-object compression and Single Arbitrarily Shaped Virtual-Object Compression using "CLAIRE" sequence (frame \#22 is shown), (a)Extracted Rectangular Virtual-Object (the colored portion only) in rectangular virtual-object compression (b) Extracted Single Arbitrarily Shaped Virtual Object (colored portion in the frame) (c) Rectangular virtual-object compression. Compression Ratio = 53:1, Average PSNR $=26.16$ (d)Single Arbitrarily Shaped VirtualObject Compression. Compression Ratio $=60.02: 1$, Average PSNR $=$ 37.12

4.5 Comparison between virtual-object compression and Single Arbitrarily Shaped Virtual-Object Compression using "CLAIRE" sequence (frame \#22 is shown), (a)Extracted Rectangular Virtual-Object (the colored portion only)in rectangular virtual-object compression (b) Extracted Multiple Arbitrarily Shaped Virtual Objects (colored portion in the frame) (c) Rectangular virtual-object compression. Compression Ratio $=53: 1$, Average PSNR $=26.16$ (d)Multiple Arbitrarily Shaped Virtual-Objects Compression. Compression Ratio $=58.19: 1$, Average $\mathrm{PSNR}=37.16$ 
4.6 PSNR comparison between rectangular virtual-object (VO), single arbitrarily shaped virtual-object (SASVO), and multiple arbitrarily shaped virtual-object (MASVO) compression methods . . . . . . . . . . . . 49

4.7 Image Sequence with multiple moving objects . . . . . . . . . . . 51

4.8 Virtual-object extraction. (a) Rectangular virtual-object (b) Single arbitrarily shaped virtual-object (c) Multiple arbitrarily shaped virtualobjects and their bounding boxes . . . . . . . . . . . . .

4.9 Performance Comparisons. (a) Rectangular virtual-object compression. Compression Ratio $=25: 1$, Average PSNR $=29.85$, (b) Single arbitrarily shaped virtual-object compression. Compression Ratio = 47.91:1, Average PSNR $=31.21$, and (c) Arbitrarily shaped multiple virtual-objects compression. Compression Ratio $=49.45: 1$, Average $\mathrm{PSNR}=31.44 \ldots \ldots \ldots \ldots \ldots \ldots \ldots$ 


\section{CHAPTER 1}

\section{INTRODUCTION}

With the advancements in multimedia technologies, effective video compression has become more and more important. With the world increasing becoming digitalized, there is a rapid increase in the already vast amount of digital images and videos. Moreover, the size of the video or image files is increasing with the increasing camera resolutions and because of never ending demand for better quality image or video signals. At the same time, there is a limit to the available storage space, hardware resources and the data transmission bandwidth. There comes the video compression into picture. Video compression is a tradeoff between the video quality, storage space and the amount of hardware resources required for encoding and decoding. Because of these many constraints, an effective compression method is of paramount importance to design a good system to store, display or transmit video. Consequently, more and more research is being carried out in the field of video compression.

The sole purpose of the video compression is to provide a compressed video that when decoded at the user's end is still of good perceptual quality. It utilizes the fact that human beings cannot distinguish between small spatial and temporal differences, and therefore, such small changes as well as spatial and temporal redundancies can be eliminated.This is lossy video compression. However, video compression can also 
be lossless if needed. Lossless video compression results in an exact bit to bit match with the original signal but is quite rarely used.

A brief overview of the various video compression standard is provided first followed by the presentation of research objectives and contributions.

\subsection{Video Compression Standards}

Video compression typically involves intra-frame coding and inter-frame coding. Intra-frame coding exploits the spatial redundancy whereas inter-frame coding exploits temporal redundancy. Moreover, depending upon the basic processing entity of compression, video compression can be broadly classified into two categories: 1) block based compression where each frame is divided into blocks before applying compres-

sion and 2) object based compression where objects of interest are extracted from the frames, and are compressed separately from the remaining background.

\section{MPEG1/MPEG2}

Moving Pictures Expert Group (MPEG) group was started in 1988 with the purpose of defining compression standards of audio-video signals. It published first such compression standard MPEG-1 in 1993 [1] followed by the publishing of MPEG-2 in 1995 [2]. In both the standards, the basis of compression is a macroblock, and therefore, these are block based compression standards. MPEG-2 is almost same as MPEG-1 with some added capabilities for higher data rates, half-pixel precision motion compensation for higher performance inter-frame prediction [2].

MPEG-2 implements intra-frame coding by dividing each frame into blocks of size 8 pixels by 8 lines, and taking 2D discrete cosine transform separately in each block, followed by quantization and variable length coding. Inter-frame coding includes 
predicting the current frame based upon the other frames in the video sequence and motion-compensation. Depending upon the nature of prediction, three frame types are defined in MPEG where frame type defines which prediction mode maybe used to code each block. Intra (I) frames are coded without reference to any other picture. Therefore, they serve as a starting point for a decoder. Moderate compression is achieved for intra frames by reducing spatial redundancy. Predictive $(P)$ frame is generated from the difference between motion-compensated current frame and the closest I frame. P frames offer better compression than I frames by exploiting spatial and temporal redundancy. Bidirectionally-predictive $(B)$ frame uses the difference between motion-compensated current frame and two closest reconstructed I frames. Highest degree of compression can be achieved for B frames.A typical coded video frames' sequence in MPEG can be of the form of:

\section{BB P BB P BB P BB P BB I ...}

Fig. 1.1 gives the design flow of the general MPEG1/MPEG2 video encoder.

MPEG-1 supports video coding up to about $1.5 \mathrm{Mbit} / \mathrm{s}$ and is used primarily for storing audio and video on a CD-ROM. For typical video sequences, MPEG-1 gives a compression ratio of around 26:1 [11]. MPEG-2 supports bit-rates of about 3$15 \mathrm{Mbit} / \mathrm{s}$ for standard definition television and supports bitrates of $15-30 \mathrm{Mbit} / \mathrm{s}$ for high-definition television. MPEG-2 gives a typical compression ratio of around 45:1 [12]. Some other compression standards using macroblocks as the basic processing entity are H261 and H263. 


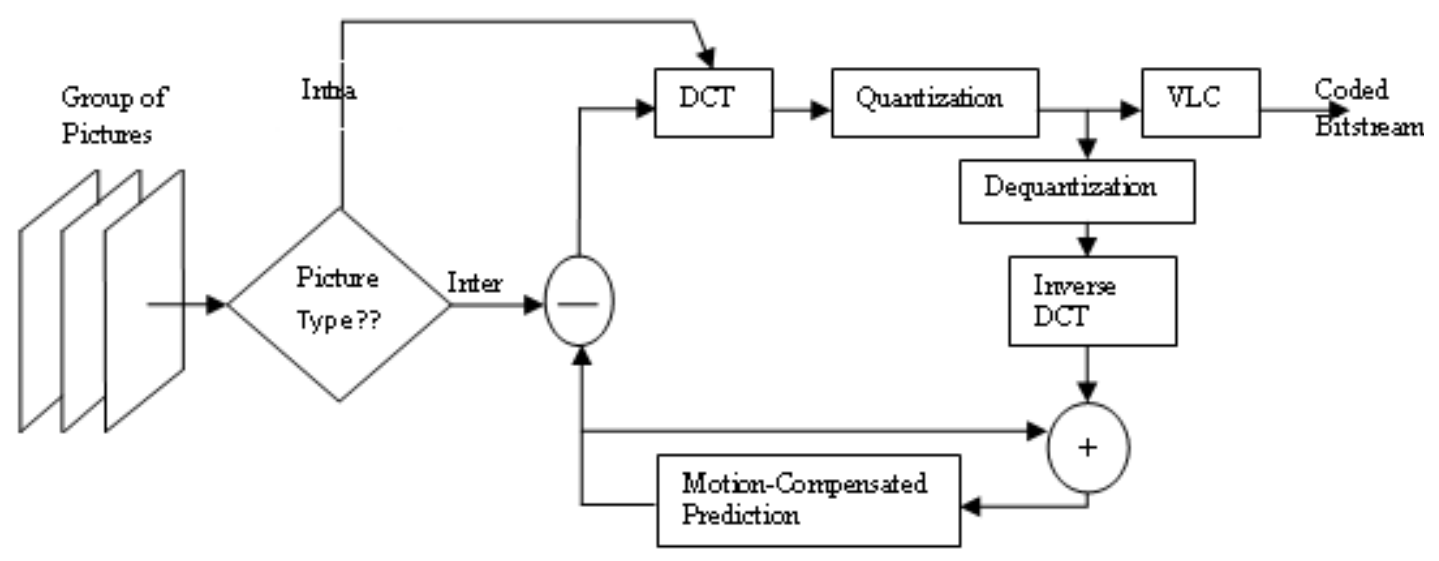

Figure 1.1: A general MPEG video encoder

\section{MPEG4}

The object based compression techniques are largely believed to be able to provide better compression than the block based techniques for the same signal quality. With this in mind, moving pictures experts group published MPEG- 4 standard [3] in 1999. Moreover, with the increasing popularity of internet and extremely higher compression needed to transmit video signals over internet because of limited bandwidth, a highly efficient compression standard was needed as MPEG-2 compression algorithms were reaching their limits.

The basis of coding in MPEG-4 is not a macroblock but rather an audio-visual object [3]. The approach separates the non-static portion of the video from the static portion, and compresses them separately. With most of the portion in a video usually being static, the method can achieve much higher compression ratio. This object based encoding is a major component of MPEG-4. 
Even though MPEG-4 specifies the syntax of encoded bit-stream and decoding procedures, significantly enough it does not provide means to separate audio-visual object from the background. This on one hand makes MPEG-4 a very much open and generic standard, and on the other hand, it has left efficient object segmentation and background generation subjected to research. Thus, MPEG-4 is a very flexible standard and various compression tools can be incorporated into it. These various compression tools are known as profiles of MPEG-4, and today there are a number of profiles available.

\section{H264}

H264 is one of the most efficient compression standards. It was developed by ITU- T Video Coding Experts Group (VCEG) together with MPEG group. For this reason, it is also known as MPEG- 4 Part 10, or MPEG- 4 AVC (Advanced video coding) [4]. H264 supports variable block size motion compensation with block sizes as large as 16X16 and as small as 4X4. Therefore, H264 can achieve precise segmentation of moving regions. Furthermore, the number of reference frames that can be used to predict current frame can be as large as 16. This achieves a significant bit-rate reduction for certain types of video scenes such as scenes with repetitive motion or uncovered background areas.

The H264 or MPEG-4 AVC is one of the most efficient coding schemes in use. It uses just 50 of the bit-rate of MPEG-2 for the same signal quality; motion estimation and compensation can be as accurate as $1 / 8$ pixel. 


\subsection{Motivations}

\subsubsection{Wavelet Compression vs Discrete Cosine Transform Compression}

The various compression methods described in section 1.1 uses Discrete Cosine Transform (DCT) to remove spatial redundancy with the exception of MPEG-4 which does not specify a compression method. The most popular video compression standard MPEG-2 uses DCT. Similarly, one of the most common image compression standards, JPEG was using DCT.

The popularity of DCT is because of its great computational performance as well as because of limited memory requirements. However, DCT is not without drawbacks. A major problem with the block-based DCT is the appearance of blocking artifacts in the reconstructed signals at low-bit rate coding. Moreover, scalable coding of image and video signals is not inherently supported as scaling must occur on per-block basis, and this does not allow the encoder to maximize fidelity across a large spatial domain. This is a major drawback as it is often required to transmit the same content at different bit-rates to different receivers as per the available bandwidth between the channels.

Over the years, significant amount of research has been conducted to find the alternatives to DCT, and wavelet transform has been found as a superior alternative to DCT for still image compression $[24,25,35]$ as well as for video compression $[21,23,31]$. Indeed a new wavelet transform based image compression standard JPEG2000 [13] has been published in March 2000, and is much superior than JPEG, the DCT based method. Wavelet transform methods have also been adopted into some profiles of MPEG-4. 
Wavelet transform does not suffer from the blocking artifacts of traditional blockbased DCT methods as it can be applied directly to the whole video frame in comparison to DCT methods where the video frame is first divided into blocks, and then DCT is applied. Moreover, wavelet decomposition of a signal results in a low resolution signal along with many finer and finer details. Thus, a wavelet decomposed signal supports continuous scalability, and encoder can select any bit-rate within a range defined by the number of levels of wavelet decomposition. Furthermore, wavelet transform localize signal characteristics in both time and frequency domains. Therefore, spatial redundancies can be efficiently exploited to achieve higher compression at a good signal quality.

In the past few years, many wavelet based compression techniques $[6,21-23,30,31]$ have been designed targeting video applications. Techniques such as 3D wavelet compression [14] utilizes wavelet transform for both spatial as well as temporal decomposition. Some other techniques uses block based motion compensation for temporal compression and wavelet decomposition for spatial compression [31]. Eric et. al proposed a wavelet based technique known as virtual-object compression [7], further improving the compression ratios possible for wavelet transform based compression. The method separates changing portion of the video from the stationary part. Changing portion of the video across various frames is grouped as a $3 \mathrm{D}$ virtual object, and compressed using 3D wavelet compression. Remaining portion of the video is compressed using $2 \mathrm{D}$ compression as it is not changing. The name virtual object comes from the fact that method extracts the rectangular object covering the changing portion in each frame rather than extracting the real object of interest. 
Virtual object compression improves the compression ratio possible for a wavelet compression method. It also provides some benefits of the true object based compression methods at a reasonable computational cost and without the extra memory overhead. However, by restricting the shape to be rectangular, lots of non-changing portion of the video is also extracted as part of virtual object and is compressed in 3D. This decreases the compression ratio possible for virtual-object method. Perhaps the shape was restricted to be rectangular because of the difficulty of applying wavelet transform to arbitrary shapes and the additional computational overhead associated with it.

In the recent years, many shape adaptive wavelet transform $[5,18,34]$ algorithm have become available. Also, lifting scheme based methods [19,29] can be utilized to further fasten the wavelet coefficients computation process. Thus, these shape adaptive wavelet transform does not add extra computational or memory overhead when compared to DCT, and it can surely give better quality pictures than DCT for the same cost. Furthermore, object based compression is receiving more and more attention these days as it is the main compression method in MPEG-4, and is believed to be superior than block based compression methods. The virtual-object compression method [7] also made an attempt to provide some of the benefits of object-based compression, and found virtual object compression to be outperforming 3D wavelet compression. Thus, object based compression methods supported by wavelet transform techniques have the potential to give the best possible compression results. Therefore, this research develops an arbitrarily shaped virtual-object compression method internally using shape adaptive wavelet transform and wavelet based compression methods. 
We provide arbitrarily shaped virtual object compression method that further enhances the compression ratio possible for virtual object approach, and also outperforms pure 3D wavelet compression. A new arbitrarily shaped virtual object concept is proposed that internally uses the shape adaptive wavelet transform techniques and 3D wavelet compression.

\subsubsection{Arbitrarily Shaped Virtual-Object Compression}

With the arrival of MPEG-4, research focus has shifted to the object based compression techniques. The basis of compression in MPEG-4 is an audio-visual object rather than the macroblock.

In the object based compression approach, visual objects of interest are extracted and coded separately from the background. The extracted object is coded in 3D to preserve the changes across frames. However, the stationary background is just compressed once in 2D as there are no changes across frames (by definition). This is what results in better compression ratio for object based compression techniques than the block based compression techniques where everything needs to be coded in 3D. However, actual object extraction is computationally too expensive making real-time compression difficult if not impossible. Eric et. al, therefore, proposed a rectangular virtual-object compression [7] technique where a rectangular area covering the changing portion of the video was extracted as virtual object rather than extracting the actual object. Inspired by the rectangular virtual-object compression method, we propose arbitrarily shaped virtual-object compression method.

As with any object based compression technique, first step is to extract the object of interest from the stationary background. However, instead of extracting the real 
objects, we focus on extracting an arbitrarily shaped virtual object which covers just the changing portion of the video. The main idea behind this is that only changing portion of the video needs $3 \mathrm{D}$ coding. Rest of the portion can be coded using 2D compression. Nondecimated temporal domain wavelet transform is used for detecting the changing portion of the video as it will give large coefficient values corresponding to changing locations. Once the arbitrary shape virtual object is extracted, it is spatially decomposed in each frame by using shape adaptive wavelet transform followed by temporal decomposition.

Experimental results show that arbitrarily shaped virtual object compression gives better compression results than the rectangular virtual-object compression for the same PSNR. Furthermore, results are comparable to MPEG-4 standard and much better than the 3D wavelet compression.

\subsection{Dissertation Overview}

The rest of the dissertation is organized as follows. In the next chapter, a brief description of shape adaptive wavelet transform is given which is used for spatial decomposition of the arbitrarily shaped object. Mainly the method proposed by [18] is discussed for an odd-symmetric biorthogonal wavelet filter which is the filter we used in our research.

Chapter 3 describes the arbitrarily shaped virtual-object compression method. First, a brief description of rectangular virtual object method is given followed by the development of arbitrarily shaped virtual object compression. We then illustrate the concept of multiple virtual objects which can further improve the compression ratio depending upon the video sequence. Following that, an attempt is made to clarify the 
importance of size of group of frames (GoF). Finally, the noising problem is explained and effect of noising is illustrated on the virtual-object extraction and compression ratio.

Chapter 4 describes the various experiments conducted to test the arbitrarily shaped virtual-object compression method. First, a comparison of the compression ratios is made between the 3D wavelet compression and arbitrarily shaped virtualobject compression methods. Following that, rectangular virtual-object and arbitrarily shaped virtual-object compression methods are compared. Experimental results show that arbitrarily shaped virtual-object compression achieves better compression ratio for the same PSNR for videos with stationary backgrounds.

Chapter 5 concludes the dissertation and provides some ideas for further improvements of arbitrarily shaped virtual object compression method. 


\section{CHAPTER 2}

\section{SHAPE ADAPTIVE WAVELET TRANSFORM}

Conventional wavelet transform methods can only be applied to rectangular shapes. However, in MPEG-4 [3], the basis of processing is an audio-visual object which can be of any arbitrary shape. With more and more research being carried out in the field of object based compression, there is a need to have methods to effectively decompose any arbitrary shape. There are already few such methods available today $[5,17,18,27]$. In this chapter, we briefly review one such method known as shape

adaptive wavelet transform $[17,18]$ which we are using extensively for our arbitrarily shaped virtual-object compression method.

\subsection{Shape Adaptive Wavelet Transform}

The shape adaptive wavelet transform for the spatial decomposition of the frames consists of mainly three steps - 1)boundary extension, 2) wavelet transform and 3) subsampling. The method is described here for odd symmetric biorthogonal wavelet filter such as a $5 / 3$ wavelet filter which is also the filter we use in our arbitrarily shaped virtual-object compression method. Other types of filter follow the same steps though with some minor differences [18]. 


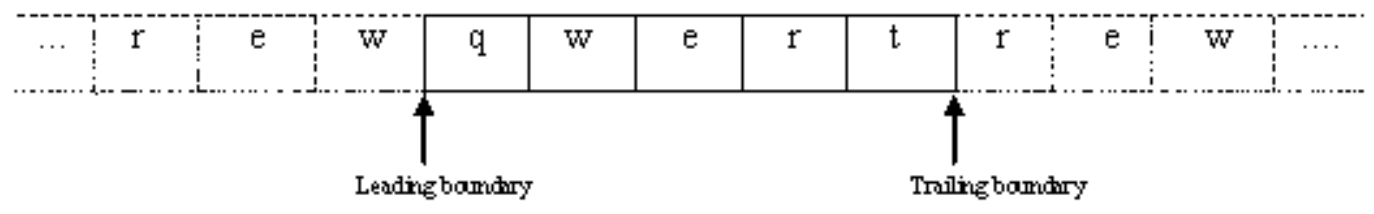

Figure 2.1: Symmetric extension for an odd symmetric biorthogonal wavelet filter at the leasing and trailing boundaries

\subsubsection{Boundary Extension}

In order to apply shape adaptive wavelet transform to a given signal, segment belonging to the arbitrarily shaped object is identified first in the given row/column for horizontal/vertical wavelet transform. The identified signal segment is then extended at the leading and trailing boundaries. The undefined pixel locations of the signal segments are filled with relevant data from inside the signal segment to maintain the perfect reconstruction property of wavelet transform. There are two types of boundary extensions - periodic, or symmetric, that can be applied to a signal/citeSAWT. The type of boundary extension needed for shape adaptive wavelet transform depends upon the wavelet filter filter type. For an odd symmetric biorthogonal wavelet filter, symmetrical signal extension is needed as it has both the low and high pass odd tap filters symmetrical about the center of the filter. As a result of symmetrical boundary extension, sharp transitions are avoided in the neighboring samples which is desirable in that it will reduce the magnitude of high-pass coefficients. Figure 2.1 demonstrates the symmetrical signal extension of a length 5 signal segment. 


\subsubsection{Arbitrary Length Wavelet Transform}

Once a signal segment is boundary extended, wavelet transform can be easily applied. To take the horizontal/vertical wavelet transform, we need to identify the segment start and the segment end in a given row/column. Moreover, there could be multiple segments in a given row/column. Each of these segments should be transformed separately.

Let the object mask of the object be denoted by $m(i)$, and let the start and end of first segment in a given row/column be defined by seg_strt (index of first 1 in object mask $m(i)$ after a 0 ) and seg_end(index of last 1 after seg_strt and before a 0 in the object mask $m(i))$ respectively. The length of the signal segment is then given by $N=$ seg_end - seg_strt +1 .

If $N=1$, the single element of the segment is repeatedly extended, and either low pass or high pass filter is applied to the signal. The decision whether to apply a low pass or high pass wavelet filter depends upon the subsampling strategy explained in the next subsection. The resulting wavelet coefficient is then placed in either low pass or high pass band depending upon which filter was applied.

If $N$ is greater than 1 , symmetrical signal extension is applied as before. To the symmetrically extended signal segment, low pass and high pass filters are applied at the alternate pixel locations starting from seg_strt and ending at seg_end. Decision about which filter should be applied first at position seg_strt depends upon the subsampling strategy. The decomposition will generate same number of low and high pass coefficients for an even length segment $(N=$ even $)$, and generates one more low pass or high pass coefficient for an odd length segment $(N=$ odd),depending upon the subsampling strategy. 


\subsubsection{Subsampling Strategy}

There are two types of subsampling strategies namely local subsampling and global subsampling that decide which pixels in the segment will be used in low pass filtering and which will be used for high pass filtering. In local subsampling, subsampling positions are decided as per the positions relative to the start of a signal segment whereas in global subsampling, subsampling positions refer to the positions relative to the beginning of the bounding box of the visual object [18]. Local even subsampling refers to the subsampling strategy where the filtering operation is applied at the even locations with respect to the start of a signal segment whereas in local odd subsampling, filter is applied at the odd positions with respect to the start of the signal segment. By locally fixing subsampling positions, we can ensure that number of low pass coefficients are always greater than or equal to the number of high-pass coefficients. However, by locally fixing subsampling position, phases of some of the low pass and some of the high-pass wavelet coefficients are skewed by one sample [18]. As a result, the local correlation among the coefficients is not preserved, and the efficiency of the wavelet transform in the second direction is degraded in case of 2D wavelet transform. Also during the decode process, the exact location of the decoded pixel can not be determined accurately. There can always be an offset of 1 from the exact location.

Fixing the subsampling positions globally, i.e. applying global subsampling strictly preserves the spatial relations and therefore, improves the efficiency of wavelet transform in the other direction. In this case, phase of the filter is fixed with respect to the global positions. Also, to achieve global even or odd subsampling, local subsampling positions have to be adjusted for each segment as starting position of each segment 
may not be always at even or odd positions relative to the bounding box [18] of the arbitrarily shaped object. For example, to achieve global even subsampling in lowpass bands and global odd subsampling in high-pass bands, local even subsampling needs to be applied in the low pass band and local odd subsampling in the high pass band if the signal segment is starting at even index with respect to the bounding box of the visual object whereas local odd subsampling needs to be applied in the low pass band and local even subsampling in the high pass band if the segment is starting at odd index with regard to the bounding box.

Wavelet decomposition with globally fixed subsampling positions will result in same number of low and high pass band coefficients if segment length is even but if segment length is odd, this can sometimes result in more number of high pass wavelet coefficients than number of low pass coefficients.

In our arbitrarily shaped virtual-object video compression method presented in next chapter, we use global even subsampling in low-pass bands and global odd subsampling in high-pass bands as such a strategy preserves the pixel locations, and results in more signal processing gain [18].

\subsection{2-D Shape Adaptive Wavelet Transform}

Based on the discussions in the above sections, 2D shape adaptive wavelet transform process can be described as below:

1. Find out the shape information of the object as well as the rectangular box enclosing the arbitrarily shaped object.

2. Using the arbitrary shape information calculated in step 1, identify the first segment of consecutive pixels in the current row. 
3. Symmetrically extend the signal segment at the leading and trailing boundaries as shown in Figure 2.1.

4. Apply the low-pass and high-pass wavelet analysis filters to the symmetrically extended segment. Low pass analysis filter is applied such that center of the filter is always at the even indices of the original segment. High pass analysis filter is applied such that its center is always at the odd indices of the original segment.

5. Store the wavelet coefficients in their respective low pass and high pass band. If within a frame/bounding box, original segment starts at index $2 i$ in a given row, the low pass as well as the high pass coefficients are stored from index $i$ onwards in their respective low pass and high pass band. On the other hand, if the original segment starts at index $2 i+1$, low pass coefficients are stored from index $i$ onwards in the low pass band whereas the high-pass coefficients are stored from index $i+1$ onwards in the high pass band.

6. Update the shape information as per the wavelet coefficients position. This is done so that we have the wavelet coefficients position information and using this information, wavelet transform in the vertical direction or at the next higher scale level can be taken.

7. Apply the steps 3-6 on the next identified segment of consecutive pixels in this row.

8. Perform the above steps for the next row of pixels. 
9. Once done with all the rows, follow the above steps are followed for each column of the 1-D transformed frame to get the 2-D transform of arbitrary shaped object.

10. Repeat all the steps as many number of times as the number of levels of wavelet decomposition desired to the low pas-low pass band of the $2 D$ decomposed object.

\subsection{Summary}

In this chapter, shape adaptive wavelet transform method presented in [17] and [18] was briefly reviewed. Various steps needed to take a 1-D wavelet transform were described in Section 2.1 specifically for an odd symmetric birothogonal wavelet filter. However, method is quite generic and can be used with other wavelet filters with slight

modifications [18]. Following the 1-D shape adaptive wavelet transform method, a step by step procedure to take 2-D wavelet transform of any arbitrary shape was presented in Section 2.2. The method is used extensively in our arbitrarily shaped virtual object compression method. 


\section{CHAPTER 3}

\section{ARBITRARILY SHAPED VIRTUAL-OBJECT VIDEO COMPRESSION}

\subsection{Introduction}

With the arrival of MPEG-4, object based video compression has received more and more attention $[7,28,34]$. In object based compression schemes, arbitrarily shaped video objects are identified, extracted, and coded separately providing new functionalities and more flexibility. More importantly, object based compression looks promising to achieve better compression while maintaining a good video quality. Therefore, with the focus shifted to object based compression methods, much research has been conducted in the area of shape coding $[16,26,36]$, texture coding $[5,10,18,20,34]$, and methods of object identification and tracking $[15,33]$.

The most important step in the object based compression is the accurate object extraction. At the same time this is the most difficult part. Given the variety of video scenes, accurate object identification as well as tracking across the frames is difficult. Moreover, usually such object extraction methods are computationally expensive, and no single method can be applied to all types of video scenes. This makes object identification and extraction in the real-time really difficult if not impossible. 
Therefore, an object based compression method that is computationally efficient as well as robust enough to work for all types of video scenes is needed. Balster et. al proposed wavelet transform based virtual object compression method where a rectangular virtual object covering the points in motion is identified and extracted instead of tracking and extracting the real objects [7]. This rectangular virtual object is compressed in three dimensions using conventional 3D wavelet compression [14]. The stationary video portion is grouped as background and coded using 2D wavelet compression. The method achieves good compression results when compared with the conventional 3D wavelet transform method. More importantly, it overcomes the difficulty in identification and tracking of real objects by restricting the object shape to be rectangular. This makes the shape coding easier and efficient but at the same time, a large part of the non-changing video portion is considered as a part of virtual object, and is compressed in 3D. As a result, compression ratio is reduced without significantly increasing the signal quality although it is still greater than the compression ratio of pure 3-D wavelet compression.

Perhaps the virtual-object shape was restricted to be rectangular in [7] as conventional wavelet transform can only be applied to rectangular frames, along with the obvious reason to make shape coding easier and faster. However, in recent years there has been significant amount of research to develop wavelet transform techniques that can be directly applied to arbitrary shapes. One such method known as Shape Adaptive Wavelet Transform was developed by Li et al. [18] and briefly described in Chapter 2. This shape adaptive wavelet transform technique works by identifying a segment of pixels in the given arbitrarily shaped object and then, transforming it after applying symmetrical extension. 
Thus, an arbitrarily shaped virtual-object compression method is presented that can provide some benefits of the object-based compression without the difficulties of real object based compression. Moreover, the method also overcomes the shortcomings of virtual object compression [7] in that it does not restrict the shape of virtual-object to be rectangular. The virtual object can be of any arbitrary shape, and method uses shape adaptive wavelet transform for spatial decomposition.

Arbitrarily shaped virtual-object compression works by separating the portion of the video exhibiting motion from the stationary portion of the video. The stationary portion of the video is grouped as background, and is compressed using 2D wavelet compression. The motion exhibiting portion of the video is grouped as arbitrarily shaped virtual object and is compressed using 3D wavelet compression. However, in $3 \mathrm{D}$ wavelet compression of the virtual-object, spatial decomposition is done using $2 \mathrm{D}$ shape adaptive wavelet transform instead of using conventional 2D wavelet transform for spatial decomposition, followed by temporal decomposition using conventional wavelet transform methods.

To separate the arbitrarily shaped virtual object from the stationary portion, nondecimated wavelet transform is applied in the temporal domain. Applying the wavelet transform in temporal domain will result in large wavelet coefficient values corresponding to locations where pixel values are changing significantly across frames. Once the wavelet coefficients are computed, a binary mask of $1 \mathrm{~s}$ and 0 s is created by applying a pre-determined motion threshold. A value of 1 indicates a pixel belonging to the moving object whereas a value of 0 indicates a pixel belonging to the stationary background. It is the binary mask which defines the shape of the virtual object in 
each frame and therefore, virtual object can be of any arbitrary shape corresponding to only the changing portion in the video.

Furthermore, idea of multiple virtual objects is presented. The rectangular virtualobject compression method proposed in [7] limits the number of virtual objects to be one for any video scene. However, a given video scene can have multiple moving objects. Moreover, if these multiple moving objects are far apart from each other in the video frame then enclosing all these moving objects in one rectangular virtual object will degrade the compression performance. In such a case, a large non-changing portion of the video is compressed in 3D as it will be considered as a part of the virtual object. Therefore, to effectively segregate the moving portion of the video from stationary background and to further improve the compression ratio, idea of multiple virtual objects is also presented.

The rest of the chapter is organized as follows. Following the introduction, section 3.2 gives a brief description of rectangular virtual-object compression [7]. Section 3.3 describes the arbitrarily shaped virtual-object compression and section 3.4 presents

the idea of multiple virtual objects. Section 3.5 discusses the importance of group of frame size followed by conclusions in section 3.6.

\subsection{Virtual-Object Video Compression}

The virtual-object video compression is explained in detail in [7]. We briefly explain it here for the purpose of completeness as well as to make understanding of our proposed concept of arbitrarily shaped virtual object easier. Virtual object based compression is an object based compression method that works by defining, as the name suggests, a virtual object instead of trying to identify and track a real object. 


\subsubsection{Virtual Object}

Virtual-object compression scheme works by separating the motion exhibiting portion from the stationary portion of the video. The portion of the video that exhibits motion is extracted as virtual object. A virtual object is defined as threedimensional rectangular object $o($.$) where o(x, y, z)$ is a pixel in the object sequence of horizontal position $x$, vertical position $y$ and frame $z$. Its dimensions are given by width $W_{o}$, height $H_{o}$, and frames $F$. Also, virtual object is restricted to be of the same size across frames so that it is easily defined and compressible. Therefore, dimensions $W_{o}$ and $H_{o}$ are constant and do not depend upon the frame number $z$.

To completely define the virtual object, horizontal and vertical positions $S_{x}[$. and $S_{y}[$.$] of the upper left corner were defined as well as the horizontal and vertical$ positions of the lower right corner as $E_{x}[$.$] and E_{y}[\cdot]$. With these definitions, there are some obvious boundary conditions. Virtual object can not be greater than the original frames, and width and height of virtual object must be positive. Therefore, $0 \leq W_{o} \leq W_{f}$ and $0 \leq H_{o} \leq H_{f}$

Thus, mathematically virtual object can be defined as:

$$
o(x, y, z)=f\left(x+S_{x}[z], y+S_{y}[z], z\right), 0 \leq x \leq W_{o}, 0 \leq y \leq H_{o}, 0 \leq z \leq F
$$

where $o($.$) is the virtual object and f($.$) is the original image sequence.$

The background corresponds to the leftover area after rectangular virtual object extraction. It is the temporal average of the entire group of frames (GoF) area outside the virtual-object boundary and is given by: 


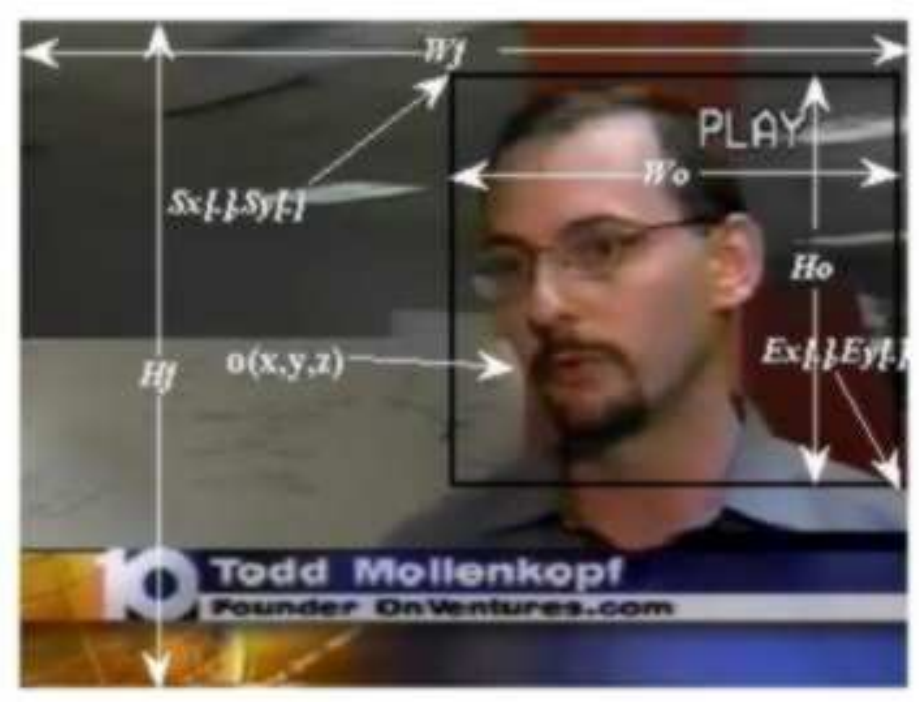

Figure 3.1: Virtual-Object Definitions

$$
b(x, y)= \begin{cases}\frac{\sum_{z=0}^{F-1} f(x, y, z) \times \alpha[x, y, z]}{\sum_{z=0}^{F-1} \alpha[x, y, z]} & \text { if } \sum_{z=0}^{F-1} \alpha[x, y, z] \neq 0 \\ 0 & \text { otherwise }\end{cases}
$$

where $\alpha[x, y, z]$ is 1 if $(x, y, z)$ belongs to a location outside the virtual object boundary, and 0 otherwise.

Figure 3.1 shows a frame of "10TV" image sequence illustrating various definitions of the virtual object.

\subsubsection{Virtual-Object Extraction}

To extract the virtual object, temporal domain wavelet transform is applied to the original video sequence $f($.$) . Applying the wavelet transform in temporal domain$ results in large wavelet coefficients at locations corresponding to the motion in the image sequence. Moreover, such wavelet coefficients are spatially contiguous [8]. 
The wavelet coefficients resulting from applying the temporal domain wavelet transform to an image sequence $f($.$) are given by$

$$
\lambda_{v o}[x, y, z]=\sum_{k} f(x, y, k) g_{v o}[k-z]
$$

where $\lambda_{v o}$ are the resulting wavelet coefficients values, and $g_{v o}$ is the wavelet filter used.

Using the resulting wavelet coefficients values, a binary mask is created identifying changing and non-changing locations in the video. The value of a wavelet coefficient is compared to pre-determined motion threshold value. If the coefficient value is greater than the threshold, corresponding pixel belongs to an object in motion, and the binary mask value at the corresponding location is set to 1 otherwise it is set to 0. Therefore,

$$
M_{v o}[x, y, z]= \begin{cases}1 & \text { if }\left|\lambda_{v o}[x, y, z]>\tau_{v o}\right| \\ 0 & \text { otherwise }\end{cases}
$$

where $M_{v o}[., ., z]$ is the resulting binary mask for each frame $z, \tau_{v o}$ is the experimentally determined motion threshold value. A better estimate can be calculated using the method described in [9].

Once the binary mask of 1 s and 0 s is found for each frame, it is further refined by applying the spatial support criteria [8]. Corresponding to resulting 3D refined binary mask, a rectangle enclosing all the 1s of the binary mask across all the frames is found [7]. This rectangle defines the starting and ending positions of virtual object for the given image sequence. It should be noted that virtual object is a 3D object - two dimensions being the dimensions defined by the rectangle enclosing all the $1 \mathrm{~s}$ 
of the binary mask, and the third dimension being the number of frames in the GoF used to extract the virtual object.

\subsubsection{Virtual-Object Video Compression}

After the virtual-object is extracted and separated from the background, virtualobject portion is compressed using the $3 \mathrm{D}$ wavelet compression method. First, spatial redundancy is exploited as virtual object portion is spatially decomposed in each frame using $2 \mathrm{D}$ wavelet transform. This is followed by $2 \mathrm{D}$ quantization to remove non-significant wavelet coefficients. As a next step, temporal redundancy is exploited by applying the temporal domain wavelet transform to the spatially decomposed virtual object portion in each frame. This is nothing but 3D wavelet transform of the virtual-object portion, and it is followed by 3D quantization, stack run encoding [32] and Huffman coding.

The background is compressed using 2D wavelet compression as only spatial redundancy can be exploited. It should be noted that corresponding to a group of frames, a single 3D virtual object and only one background frame are extracted. By definition background is the non-changing portion of the video and therefore, does not change across the group of frames. Thus, only one background frame needs to be sent for a given group of frames.

Figure 3.2 gives the overall flow of the virtual-object compression method.

\subsubsection{Virtual-Object Video Compression Results}

For a given video sequence, virtual-object compression method should give better compression result than the 3D wavelet compression. In $3 \mathrm{D}$ wavelet compression, 


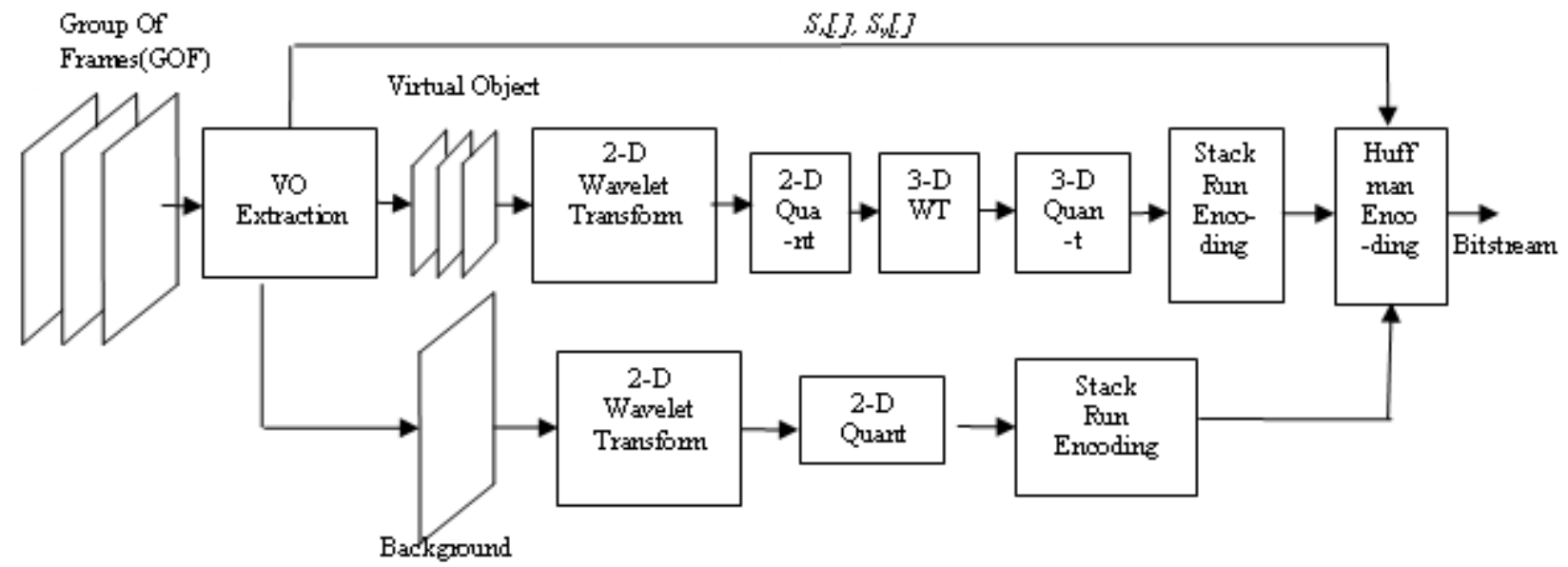

Figure 3.2: Virtual-Object Video Compression

the video sequence is compressed by applying $3 \mathrm{D}$ wavelet transform across all the frames over all the portion of the individual frame whereas in virtual-object compression, 3D wavelet transform is applied only to the virtual-object portion. Therefore, virtual-object compression can better utilize the temporal redundancy by sending the stationary portion as just one background frame for a given GoF. Figure 3.3 gives the result of performance comparison between virtual-object and 3-D wavelet compression for "10TV" image sequence. Clearly virtual-object video compression outperforms 3D wavelet compression. The GoF size was 64 in both the cases.

\subsection{Arbitrarily Shaped Virtual Object Compression}

Although the virtual-object video compression gives us good compression results compared to 3D wavelet compression, it does not exploit the temporal redundancy 


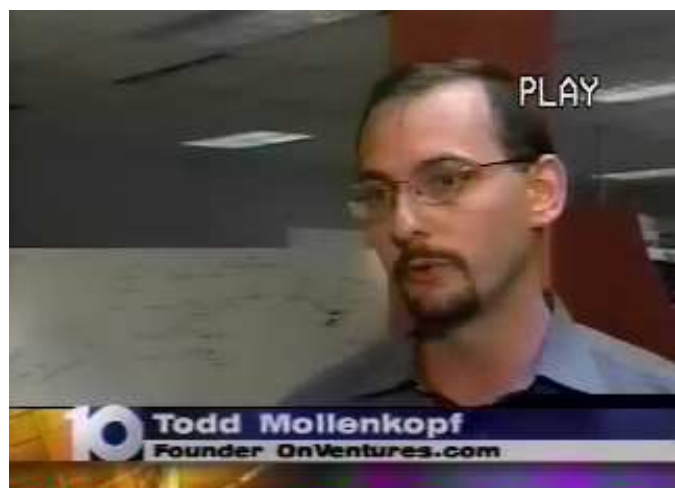

(a)

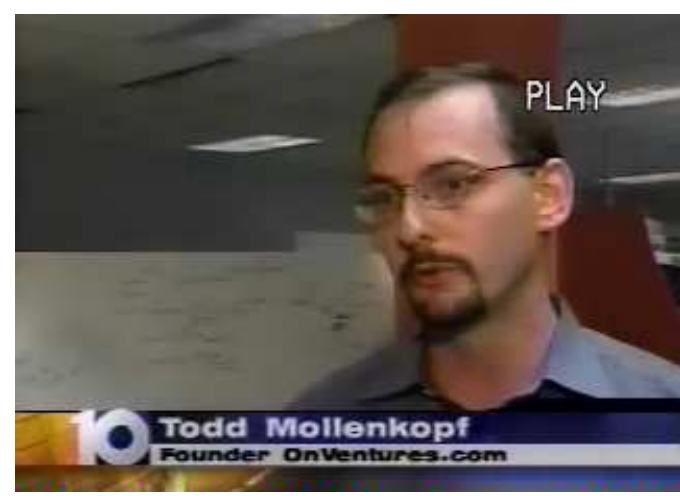

(b)

Figure 3.3: Comparison between 3-D compression and Virtual-Object Compresson using "10TV" sequence (frame \#22 is shown), (a) 3D wavelet compression. Compression ratio $=36: 1$, Average PSNR $=31.12$, (b) Virtual-Object compression. Compression Ratio $=40: 1$, Average PSNR $=31.16$

to the maximum extent possible. Therefore, arbitrarily shaped virtual-object compression method is developed to further exploit temporal redundancy, and increase compression ratio.

As stated in subsection 3.2.3, only one background frame, which is the temporal average of pixel values at locations outside the virtual object boundary, needs to be sent for a group of frames. As the area outside virtual object boundary does not change, it can be closely approximated by the temporal average of pixel values. This background frame just needs $2 \mathrm{D}$ compression. As a result, better compression ratio can be achieved compared to pure 3D compression because a lesser number of wavelet coefficients need to be encoded. Thus, separating the non-changing portion from the changing portion of the video is the key to good compression.

However, as the shape of the virtual-object is restricted to a rectangular shape, not always background can be separated effectively from the moving portion of the 
video. In fact, a significant non-changing portion of the video gets extracted as a part of the $3 \mathrm{D}$ rectangular virtual object resulting in a decrease in the compression ratio. Situation gets worse if some object happens to be moving across the diagonal of the frames in which case whole frame will become the virtual object, and the compression method will degrade to a 3D video compression method, decreasing the compression ratio. Similarly, if there are multiple moving objects in the video scene, the rectangle enclosing all the objects will also cover a lot of the non-changing portion of the video, and again best possible compression ratio can not be achieved.

Therefore, we present arbitrarily shaped virtual-object based compression method that overcomes the shortcomings of the rectangular shaped virtual object by allowing the virtual-object to take any arbitrary shape.

\subsubsection{Arbitrarily Shaped Virtual-Object}

Arbitrarily shaped virtual object corresponds to the portion of the video which is changing and needs 3D compression. As it could of any arbitrary shape, it is defined by a binary object mask of $1 \mathrm{~s}$ and 0 s where a value of 1 means the corresponding location is the part of the virtual object. The dimensions of the object mask are same as that of the frame given by width $W_{f}$, and height $H_{f}$ as before.

Let us define $f($.$) as an image sequence of a video scene. To determine the$ arbitrarily shaped virtual object, 3D nondecimated wavelet transform is applied in the temporal domain given by

$$
\psi_{\text {avo }}[x, y, z]=\sum_{k} f(x, y, k) g_{a v o}[k-z]
$$


where $\psi_{\text {avo }}$ are the resulting coefficient values, and $g_{\text {avo }}$ are the wavelet filter coefficients

Haar Wavelet function is used to determine the arbitrarily shaped virtual-object because of its compact support, and a well known fact that biorthogonal Haar wavelet function gives the best results for motion identification. In fact, applying the Haar wavelet filter is just equivalent to a simple pixel to pixel difference between consecutive frames. Therefore, spatial and temporal location of the motion can be easily located by testing the wavelet coefficients' values against a motion threshold.

A 3D binary mask determining motion from non-motion is computed by applying a motion threshold check on the wavelet coefficient values:

$$
T M_{\text {avo }}[x, y, z]= \begin{cases}1 & \text { if }\left|\psi_{\text {avo }}[x, y, z]>\tau_{\text {avo }}\right| \\ 0 & \text { otherwise }\end{cases}
$$

As with the rectangular virtual-object approach, mask is further refined by applying the spatial support criteria defined in [8]. Therefore,

$$
B M_{\text {avo }}[x, y, z]= \begin{cases}1 & \text { if }\left|S_{\text {avo }}[x, y, z]>s_{\text {avo }}\right| \\ 0 & \text { otherwise }\end{cases}
$$

where $S_{\text {avo }}[$.$] and s_{\text {avo }}$ are determined from $T M_{a v o}[$.$] using the method given in [9].$

Resulting 3D refined binary mask provides information about where a pixel value in a given frame is changing significantly from its previous frame in the group of frame. These significantly changing locations need to be subjected to 3D compression across the whole GoF to preserve temporal domain changes. Therefore, if the value 1 appears in $B M_{a v o}$ at a location specified by $[x, y, z]$, pixel values at corresponding location $[x, y]$ are transformed in the temporal domain irrespective of frame number $z$. Complete information of the points needing 3D compression, or the $2 \mathrm{D}$ shape of 
the arbitrarily shaped virtual object in each frame is therefore given by a 2D mask $O M_{\text {avo }}[x, y]$ which is computed simply by applying logical OR operation to the $3 \mathrm{D}$ mask $B M_{a v o}$ across the z-direction as:

$$
O M_{a v o}[x, y]= \begin{cases}1 & \text { if for the same }[x, y], B M_{a v o}[x, y, z]=1 \text { for any } \mathrm{z} \\ 0 & \text { otherwise. }\end{cases}
$$

Thus, object mask is a $2 \mathrm{D}$ mask giving the pixel locations that belong to the arbitrarily shaped virtual object in each frame. Again, it should be noted that virtual object is a $3 \mathrm{D}$ object with the object mask defining $2 \mathrm{D}$ arbitrary shape of the 3D object in each frame, the third dimension being the number of frames in the current group of frame.

Using the binary shape mask information, arbitrarily shaped virtual-object can be defined as:

$$
A V O(x, y, z)=f(x, y, z) \text { iffOM } M_{a v o}[x, y]=1
$$

where $A V O(x, y, z)$ is the $3 \mathrm{D}$ arbitrarily shaped virtual object corresponding to the image sequence $f(x, y, z)$ with total number of frames being $F$, and $0 \leq x<W_{f}$, $0 \leq y<H_{f}$, and $0 \leq z<F$.

The 2-D background $b_{\text {avo }}(x, y)$ is defined as:

$$
b_{\text {avo }}(x, y)= \begin{cases}\frac{\sum_{z=0}^{F-1} f(x, y, z) \times \alpha[x, y, z]}{\sum_{z=0}^{F-1} \alpha[x, y, z]} & \text { if } \sum_{z=0}^{F-1} \alpha[x, y, z] \neq 0 \\ 0 & \text { otherwise }\end{cases}
$$

where

$$
\alpha[x, y, z]= \begin{cases}1 & \text { iff } O M_{a v o}[x, y]=0 \text { for any } z \\ 0 & \text { iff } O M_{a v o}[x, y]=1 \text { for any } z\end{cases}
$$




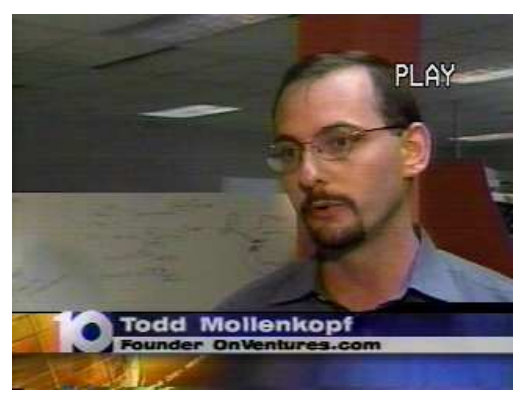

(a)

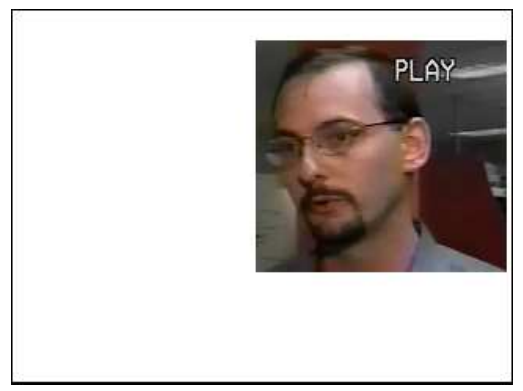

(b)

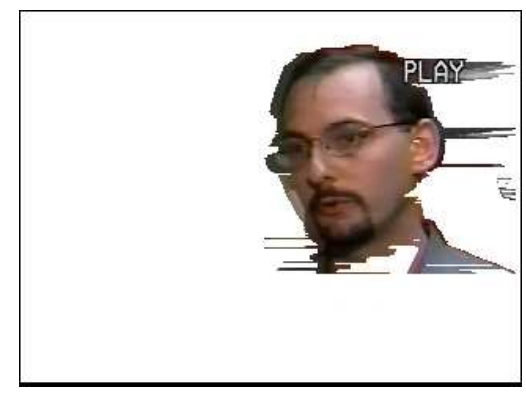

(c)

Figure 3.4: Arbitrarily Shaped Virtual-Object Extraction(frame \#22 of "10TV" image sequence is shown). (a) Original Frame, (b) Rectangular Virtual-Object, and (c) Arbitrarily Shaped Virtual-Object. Note that the stationary background is intentionally shown white for clarity purpose. Only the colored portion shown in (b) and (c) belongs to the virtual-object and is compressed in 3D. As colored portion is much less in (c), compression ratio will be higher in arbitrarily shaped virtual-object compression method

Figure 3.4 shows an original frame of "10TV" sequence, the rectangular virtual-object and the new proposed arbitrarily shaped virtual-object.

\subsubsection{Arbitrarily Shaped Virtual-Object Video Compression}

Object mask computation defines arbitrarily shaped virtual-object region as well as the background. The background is compressed using conventional 2D wavelet compression where first $2 \mathrm{D}$ wavelet transform of the background is taken followed 
by $2 \mathrm{D}$ quantization, stack-run encoding and Huffman coding. The arbitrarily shaped virtual object compression uses 3D wavelet compression method with one significant difference.

In 3D wavelet compression, spatial decomposition of the frame is done using conventional wavelet transform, one that can only be applied to rectangular frames. However, in the method presented here, conventional 2D wavelet transform can not be applied as the virtual object is of arbitrary shape. Therefore, shape adaptive wavelet transform technique needs to be used briefly described and in more detail in $[5,17,18]$. Spatial decomposition of the arbitrarily shaped virtual object using shape adaptive wavelet transform technique in each frame is followed by $2 \mathrm{D}$ quantization, temporal domain decomposition, 3D quantization, stack run encoding and Huffman coding.

In addition to background and virtual-object coding, shape coding of the virtual object or the binary mask coding also needs to be done to enable the decoder to correctly decode virtual-object and the background. As the shape information is just a binary mask of $1 \mathrm{~s}$ and $0 \mathrm{~s}$, it is directly subjected to stack run encoding followed by Huffman coding. Figure 3.5 shows the design flow of the basic arbitrarily shape virtual-object compression method.

\subsubsection{Number of Virtual Objects}

Arbitrarily shaped virtual object extraction identifies the significantly changing pixels across the group of frames. Spatial decomposition of such pixels is done using 2D shape adaptive wavelet transform in each frame. As stated in Chapter 2 and 


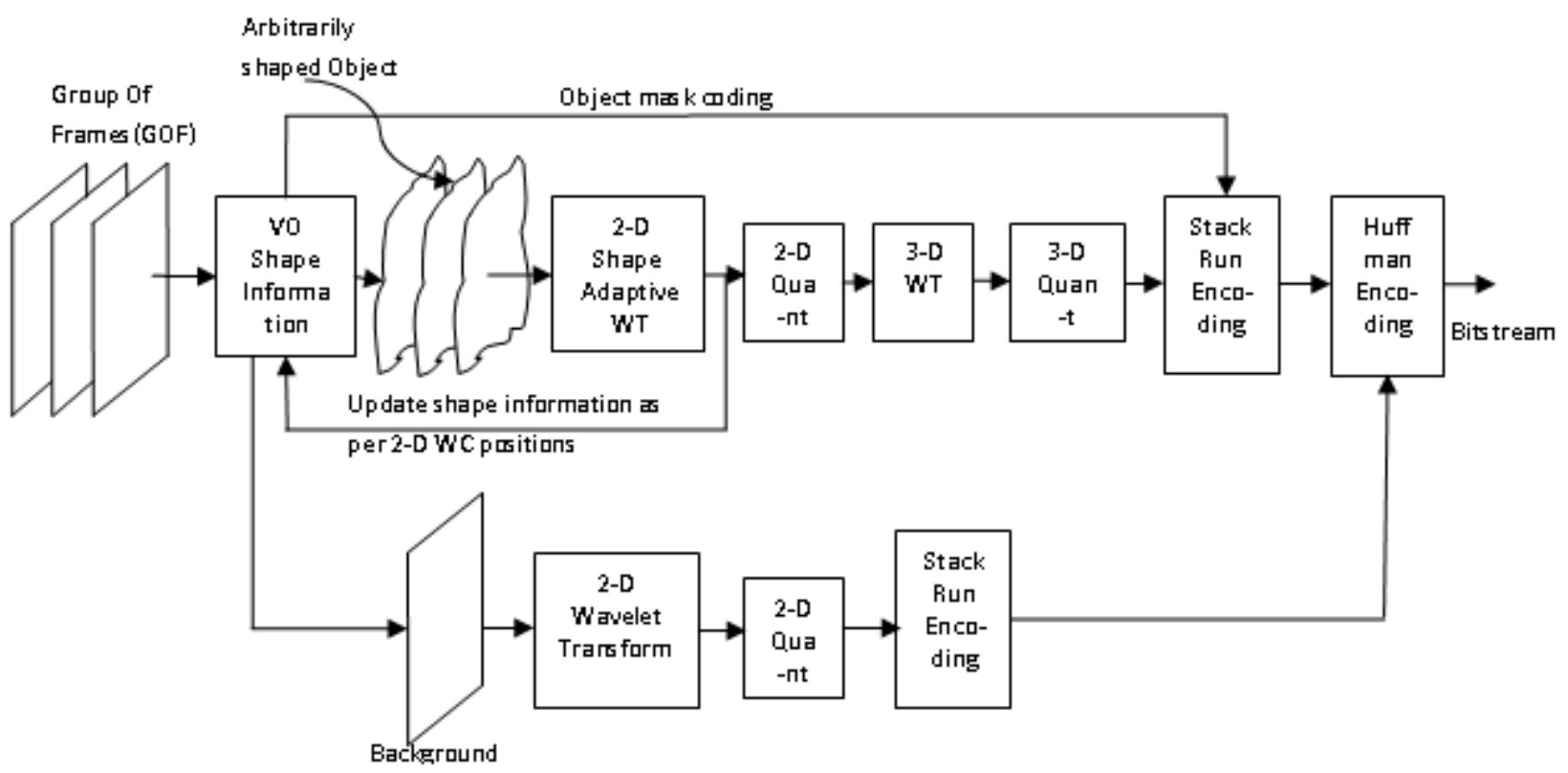

Figure 3.5: Arbitrarily Shaped Virtual-Object Video Compression

discussed in detail in [18], a rectangular box enclosing the arbitrarily shaped object is needed to compute the position where a given wavelet coefficient should be stored (to define the subsampling strategy). There are many ways to find this bounding box. Number of such bounding boxes defines number of arbitrarily shaped virtual objects. Accordingly, there can be two types of compression mechanism - single arbitrarily shaped virtual object video-compression mechanism, and multiple arbitrarily shaped virtual objects video-compression mechanism. Each of these methods has their own advantages and disadvantages which are discussed here in this subsection.

1) Single Arbitrarily Shaped Virtual Object Video Compression: This is the most straightforward method where the frame itself can be considered as a bounding box. The arbitrary shaped defined by the object mask is considered to be belonging to a 
single object bounded by the frame boundaries, and hence the name single virtualobject video compression. No additional computations are needed to compute the bounding box in this case, and the global subsampling positions to apply wavelet filters [18] are also easily defined with respect to the start of the row/column of the original frame. For example, to achieve global even subsampling in low-pass bands and global odd subsampling in high-pass bands, local even subsampling needs to be applied in the low pass band and local odd subsampling in the high pass band if the signal segment is starting at even index with respect to the start of row/column whereas local odd subsampling needs to be applied in the low pass band and local even subsampling in the high pass band if the segment is starting at odd index with respect to the start of row/column. Figure 3.5 is basically the design-flow of an arbitrarily shaped single virtual object video-compression.

There are few problems with this approach however. In a given video sequence, there could be many moving real objects. Corresponding to these moving objects, the extracted object mask will have clusters of 1 s at positions belonging to these moving objects. Enclosing all these clusters of 1 s (different moving objects) by a single rectangular box and then carrying out the shape adaptive spatial decomposition will result in high pass band coefficients with relatively large magnitudes, and in low pass band coefficients with relatively large differences in magnitude. At the next level of wavelet decomposition, this will again result in relatively large high-pass coefficient values and so on. As a result, in case of video scenes with multiple moving objects, the coding efficiency will be degraded and the compression ratio could be slightly reduced. 


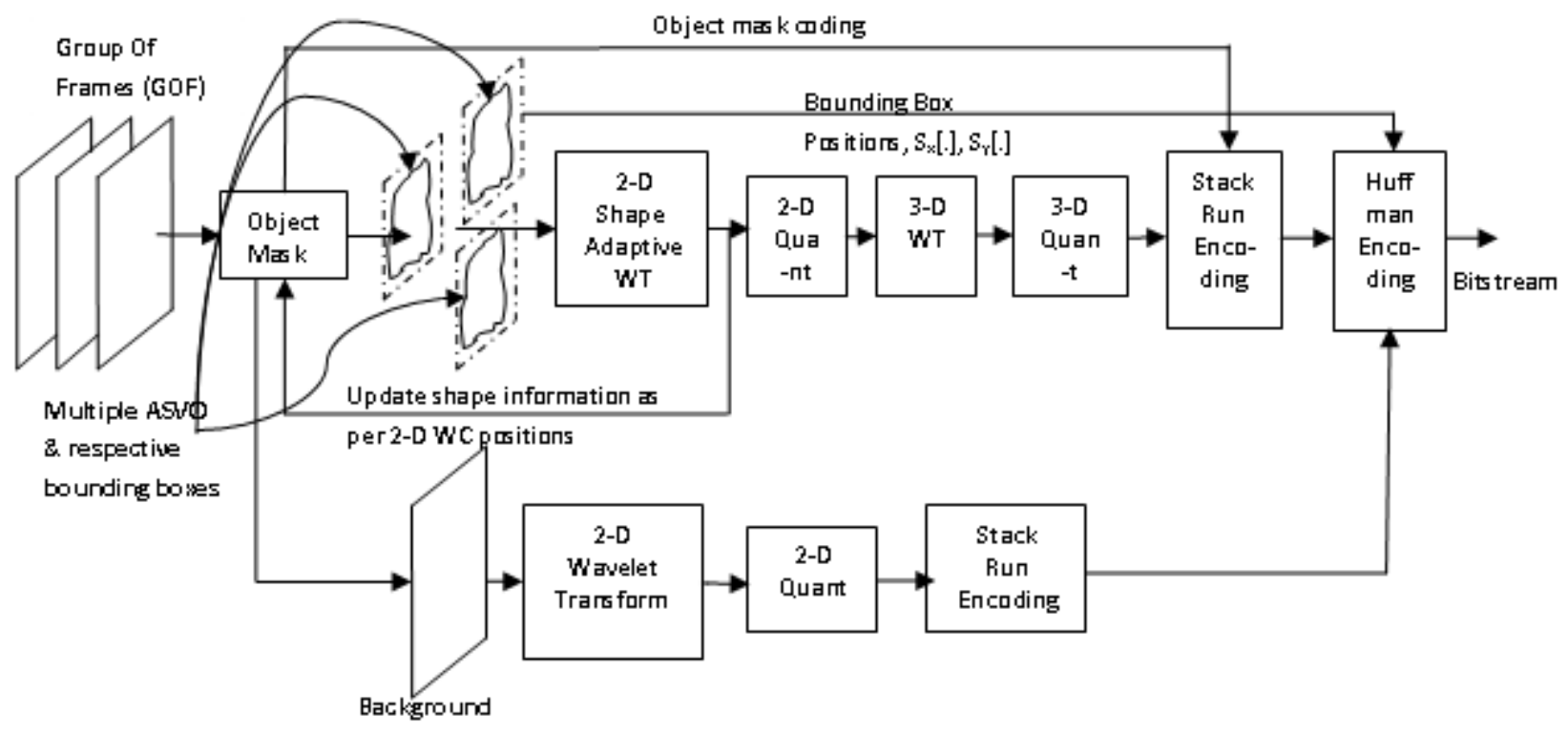

Figure 3.6: Multiple Arbitrarily Shaped Virtual-Objects Video Compression

2) Multiple Arbitrarily Shaped Virtual Objects Video Compression: In arbitrarily shaped multiple virtual-objects video compression mechanism, all the 1s of the object mask are not enclosed inside a single rectangular box. Instead an attempt is made to enclose all different objects in different bounding boxes. Usually, multiple moving objects in the video sequence will result in multiple clusters of $1 \mathrm{~s}$ in the object mask. As discussed above, if all these different clusters of 1s corresponding to different objects are enclosed within a single rectangle, coding efficiency will be degraded. At the same time, a bounding box is needed to define subsampling strategy for shape adaptive wavelet transform. Therefore, to effectively distinguish objects from one another to increase coding efficiency, and to facilitate shape adaptive wavelet transform, a different bounding box is found corresponding to each cluster of $1 \mathrm{~s}$ in the 
object mask. It should be noted that the bounding box boundaries just separates one arbitrarily shaped virtual object from the other arbitrarily shaped virtual object, and defines subsampling locations for low-pass and high-pass filters; the shape of the virtual object is still arbitrary defined by the object mask values within each bounding box. Figure 3.6 shows the design flow of multiple arbitrarily shaped virtual object video compression.

Though the arbitrarily shaped multiple virtual objects video compression achieves better coding efficiency in case of a video scene with multiple moving objects, it is having additional computational overhead of determining the positions of multiple bounding boxes. Moreover, along with the object mask of the virtual object, starting and ending positions of the bounding boxes need to be coded and stored so that the decoder side can place the decoded pixels at their correct positions. This coding of the bounding box positions will increase the compressed file size and therefore, it will contribute to an increase in the compressed file size decreasing the compression ratio. On the other hand, coding different objects separately will result in an increase in coding efficiency as result of lesser magnitude of the high-pass band coefficients' values. This will contribute to a decrease in compressed file size increasing compression ratio. Therefore, depending upon the scene type, compression ratio will either increase or decrease. In general, for a video scene with single object, single arbitrarily shaped virtual-object compression will give better result because of no additional overhead of coding bounding boxes' positions. Similarly, multiple arbitrarily shaped virtual-objects compression gives better compression results for a video scene involving multiple moving objects because the additional coding efficiency gain usually is more than the additional overhead of coding bounding boxes' positions. 


\subsubsection{Group of Frames}

Arbitrarily shaped virtual object extraction works by finding out the object mask from a group of frames $(\mathrm{GoF})$. The size of the GoF greatly impacts the compression performance, and this is what we discuss here in this subsection.

As stated before, a single background frame needs to be sent for every GoF. Therefore, it seems obvious to choose a large GoF size to achieve best possible compression ratio. On the other hand, the shape of the arbitrarily shaped virtual object is given by a $2 \mathrm{D}$ mask which is computed simply by applying logical OR operation to the 3D mask $B M_{\text {avo }}[x, y, z]$ across the z-direction over the GoF and is given by:

$$
O M_{\text {avo }}[x, y]= \begin{cases}1 & \text { if for the same }[x, y], B M_{a v o}[x, y, z]=1 \text { for any } \mathrm{z} \\ 0 & \text { otherwise. }\end{cases}
$$

Therefore, larger the number of frames in a GoF, large is the probability of finding a change in the temporal domain resulting in more number of $1 \mathrm{~s}$ in the object mask and hence, a larger virtual object. In general for a given video sequence, increasing the number of frames in the GoF will result in a decrease in the number of background frames needed to be sent thereby increasing the compression ratio. At the same time, increasing the number of frames in the GoF will result in an increase in the virtual object size thereby decreasing the compression ratio. Therefore, there is a tradeoff between the compression ratio and the size of the group of the frame. Thus, the GoF size needs to be carefully selected to achieve the best possible compression ratio for a given video sequence.

It should also be noted that the group of the frame size depends upon the video sequence too. If the video sequence is quite stable without many moving objects, large number of frames can be grouped together with not much increase in the size of 
arbitrarily shaped virtual object. In such a case, compression ratio will be increased by increasing the size of GoF as just one background frame needs to be sent corresponding to a large number of frames with very small increase in the virtual object size.

On the other hand, only a small number of frames should be grouped together if the video is having a large number of moving objects in a stationary background. This is because grouping large number of frames together in such a case will considerably increase the virtual object size with not so much savings from just sending one background as the background area will be very small. For example in a traffic surveillance video, object could be in one portion of the frame, and at the very next moment it could be at some other portion of the frame. With multiple such objects and a large GoF size, object mask will be 1 at most of the location resulting in a large virtual object and decreased compression ratio. In the worst case, a large GoF size and multiple moving objects in the video can degenerate the arbitrarily shaped virtual-object compression to 3D wavelet compression. Therefore, GoF size should not be a very large value.

Furthermore, every system has its own memory constraint which should be taken care of. A larger GoF needs more memory to store all the frames and therefore, one can not increase the GoF size endlessly even if the video is not changing significantly. Therefore, a practical upper limit defined by memory constraints is set upon the GoF size. Actual GoF size is found by simple pixel to pixel difference across frames. Starting from the first frame, difference is taken across consecutive frames. As long as the sum of differences across frame is less than a pre-determined threshold value and the total number of frames in the current GoF is less than the upper limit defined 
by memory constraint, new frame is not much different than the previous frame, and is added to the GoF containing the previous frame.

Once the GoF is changed, object mask is computed again, previous background frame is discarded, and overall arbitrarily shaped virtual object compression mechanism is repeated for the new GoF.

\subsection{Conclusions}

In this chapter, a new object based compression method called arbitrarily shaped virtual-object compression has been presented. The method differs from the conventional compression methods in that it does not use any block based compression technique. Instead, the newly presented compression method separates the arbitrarily shaped changing portion from the non-changing portion of the video, and then both are coded independently using wavelet compression methods. This arbitrarily shaped virtual-object extraction, and independent coding of the object and background results in better compression than the 3D wavelet compression and the rectangular virtual-object compression. Additionally, arbitrarily shaped virtual-object compression provides some of the benefits of the object based compression at a relatively low computational cost. 


\section{CHAPTER 4}

\section{EXPERIMENTS AND RESULTS}

Arbitrarily shaped virtual-object compression method is compared to the 3D wavelet compression and to the rectangular virtual-object compression. In addition, it is also compared to the latest compression standards such as MPEG-4 and H264. Results are provided for both the single arbitrarily shaped virtual-object compression as well as for multiple arbitrarily shaped virtual-object compression. Compression ratio has been used as a measure of performance for a given PSNR.

\subsection{Performance Comparison between 3D Wavelet and Ar- bitrarily Shaped Virtual-Object Compression \\ 4.1.1 Single Arbitrarily Shaped Virtual Object}

Single arbitrarily shaped virtual-object compression is compared to 3D wavelet

compression. The "10TV" sequence is used for comparison. Figure 4.1 gives the compression results using $10 \mathrm{TV}$ sequence. Note that for the results given, the GoF size was set to 16 frames.

Clearly, single arbitrarily shaped compression method gives better results than the 3D wavelet compression. The compression ratio achieved using single arbitrarily shaped 


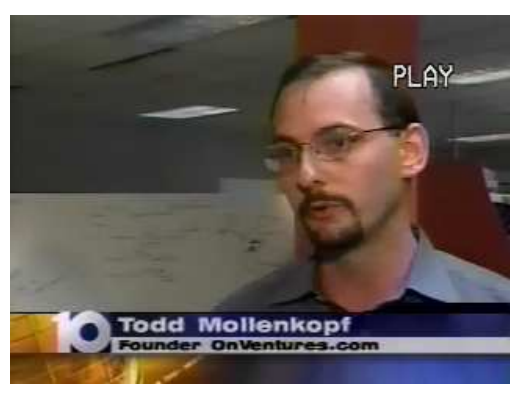

(a)

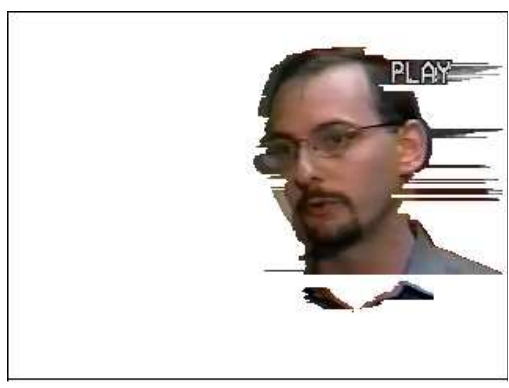

(b)

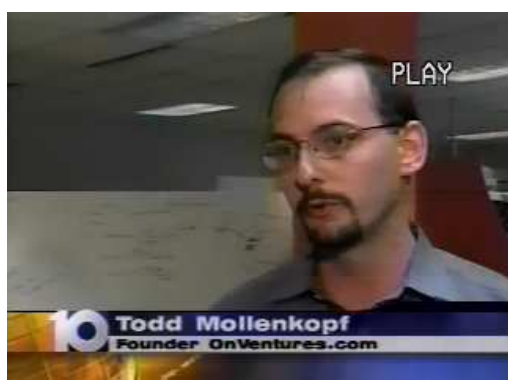

(c)

Figure 4.1: Comparison between 3-D compression and Single Arbitrarily Shaped Virtual-Object Compression using "10TV" sequence (frame \#22 is shown), (a) 3D wavelet compression. Compression ratio $=23: 1$, Average PSNR $=31.53$, (b) Extracted Arbitrarily Shaped Virtual Object, the whole colored region is considered to be belonging to the same single virtual object and is bounded by single rectangular box of dimensions same as the frame size ( background intentionally shown white for clarity) (c) Single Arbitrarily Shaped Virtual-Object Compression. Compression Ratio $=42.22: 1$, Average PSNR $=33.83$

virtual-object compression is much higher than the $3 \mathrm{D}$ wavelet compression along with an increase in PSNR value.

\subsubsection{Multiple Arbitrarily Shaped Virtual Object}

Multiple arbitrarily shaped virtual-object compression is also compared to 3D wavelet compression. Again the "10TV" sequence is used for continuity with the 


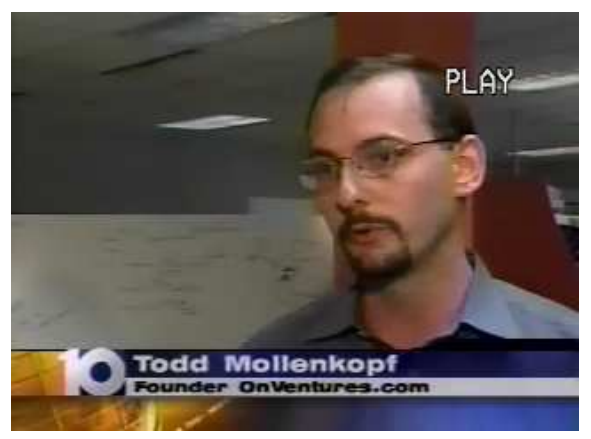

(a)

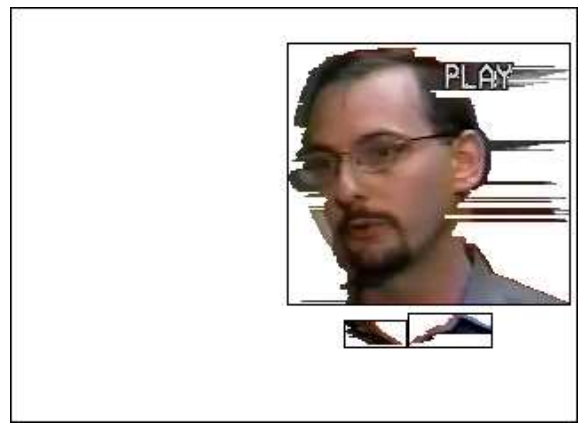

(b)

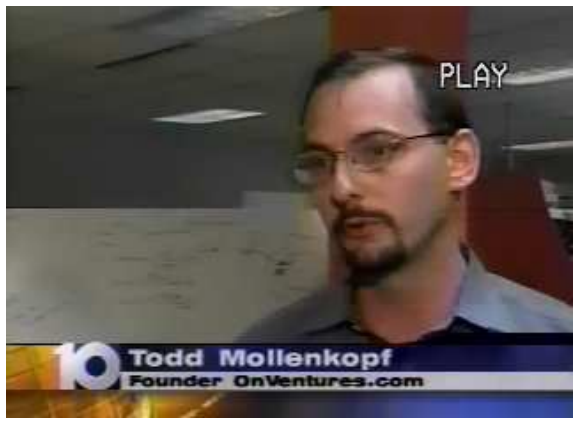

(c)

Figure 4.2: Comparison between 3-D compression and Multiple Arbitrarily Shaped Virtual-Object Compression using "10TV" sequence (frame \#22 is shown), (a) 3D wavelet compression. Compression ratio $=23: 1$, Average PSNR $=31.53$, (b) Extracted Multiple Arbitrarily Shaped Virtual Objects along with their bounding boxes ( background intentionally shown white for clarity) (c) Multiple Arbitrarily Shaped Virtual-Object Compression. Compression Ratio $=47: 1$, Average PSNR $=33.76$

comparison of 3D wavelet compression to the single arbitrarily shaped virtual-object compression . Figure 4.2 gives the compression results using $10 \mathrm{TV}$ sequence for a GoF size of 16 .

Therefore, multiple arbitrarily shaped compression method clearly outperforms the 3D wavelet compression. The compression ratio achieved using multiple arbitrarily shaped virtual-object compression is much higher than the 3D wavelet compression 
along with an increase in PSNR value. Moreover, compression ratio achieved is even higher than the compression ratio of single arbitrarily shaped virtual-object compression although there is a very slight decrease in PSNR value.

In addition to the "10TV" sequence, arbitrarily shaped virtual-object compression was applied to the "CLAIRE" and "AKIYO" image sequences. A comparison was made with the 3D compression. The result of the comparison in term of decompressed signal quality and compressed file sizes are given in Figure 4.3. Note that for all these tests GoF size was set to 16 frames. Results clearly indicate that arbitrarily shaped virtual-object compression consistently outperforms 3D wavelet compression. In addition, single arbitrarily shaped virtual-object compression gives result very much similar to the multiple arbitrarily shaped virtual-object compression. This is understandable as all these video sequences are having just one moving object. Therefore, applying multiple arbitrarily shaped virtual-object compression will slightly decrease the compression ratio because of extra overhead of coding the bounding box positions and because of coding parts of the same objects separately.

\subsection{Performance Comparison between Rectangular Virtual- Object and Arbitrarily Shaped Virtual-Object Compres- sion}

The arbitrarily shaped virtual-object method is compared to the rectangular virtual-object for both the single arbitrarily shaped virtual-object case as well as for the multiple arbitrarily shaped virtual-object case. The following subsections demonstrate the comparison results. 


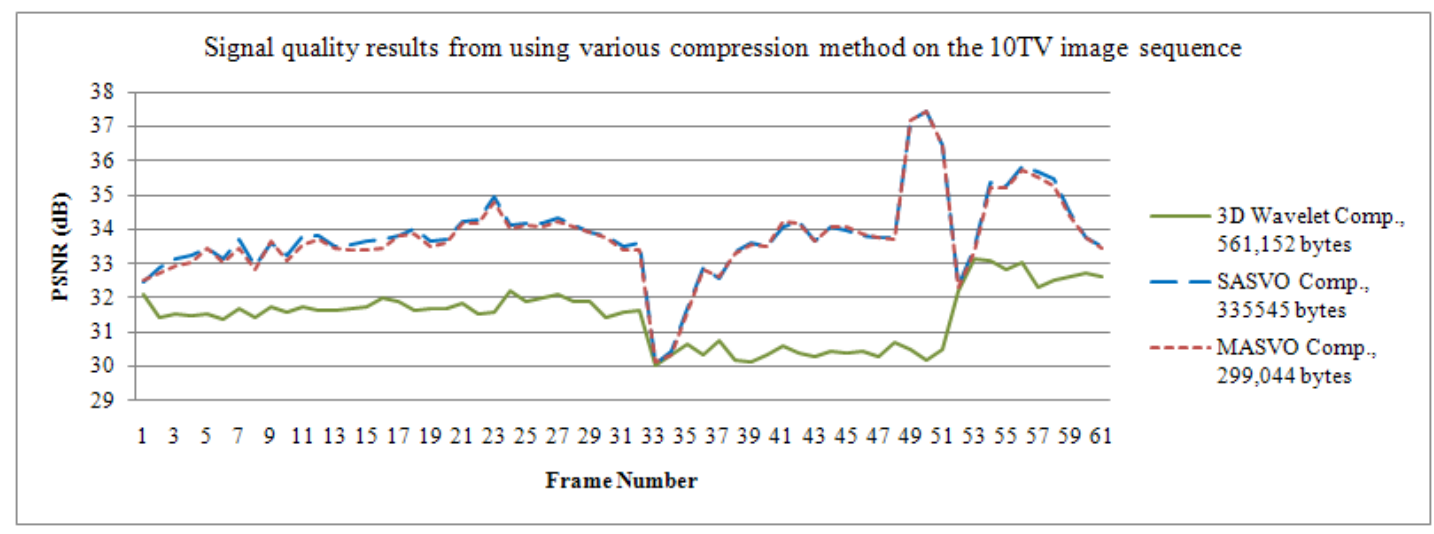

(a)

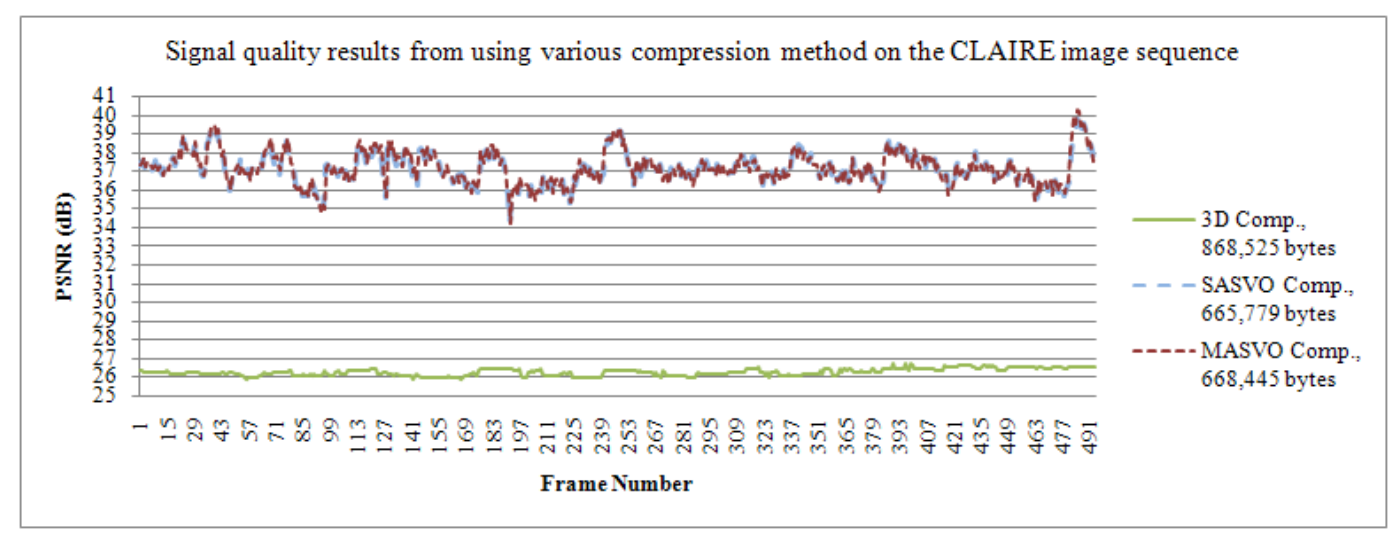

(b)

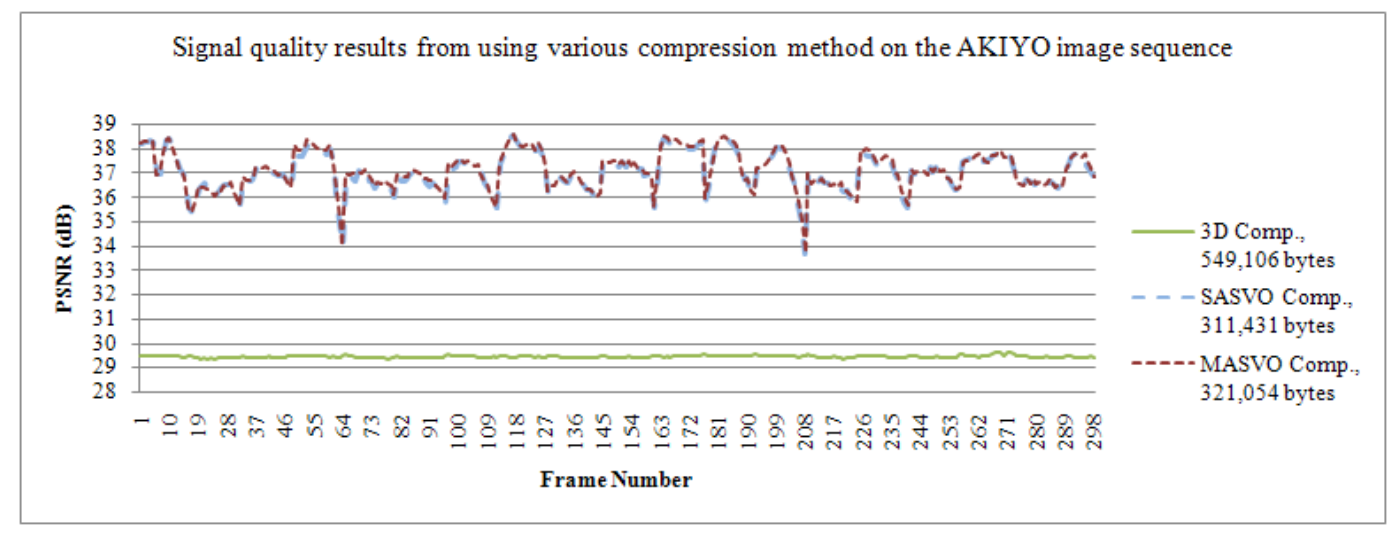

(c)

Figure 4.3: PSNR comparison between 3D wavelet, single arbitrarily shaped virtualobject (SASVO), and multiple arbitrarily shaped virtual-object (MASVO) compression methods 


\subsubsection{Single Arbitrarily Shaped Virtual Object}

Single arbitrarily shaped virtual-object compression is compared to rectangular virtual-object compression [7]. If we use the "10TV" sequence, rectangular virtualobject compression results are same as those reported for pure 3D wavelet compression in subsections 4.1.1 and 4.1.2. This is because, rectangular virtual-object extracted for the "10TV" video sequence covers the whole frame and as a result, rectangular virtual-object compression degrades to a pure 3D wavelet compression. Therefore, we use "CLAIRE" sequence in this case for better comparison with rectangular virtualobject method. Figure 4.4 gives the compression results using "CLAIRE" sequence. Note that for the results given, the GoF size was set to 16 frames.

Thus, arbitrarily shaped virtual-object compression gives better compression ratio and at the same time, a higher PSNR value. Therefore, it outperforms rectangular virtual-object video compression.

\subsubsection{Multiple Arbitrarily Shaped Virtual Object}

Additionally, multiple arbitrarily shaped virtual-object compression is also compared to rectangular virtual-object compression for the "CLAIRE" sequence. As "CLAIRE" sequence is not having multiple objects and therefore, no improvement over single arbitrarily virtual-object compression is expected. However, the method outperforms rectangular virtual-object compression as expected: the compression ratio achieved and the resulting PSNR values are much higher than those for rectangular virtual-object compression. Furthermore, the values are very similar to those of single arbitrarily shaped virtual-object compression reported in subsection 4.4. Figure 4.5 


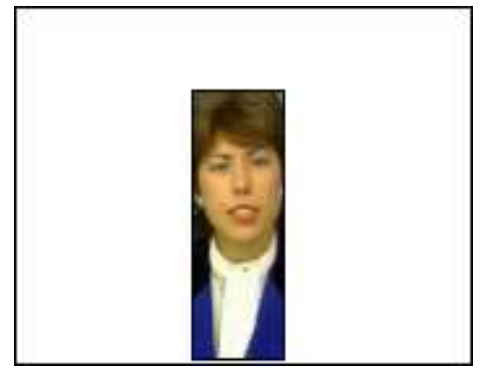

(a)

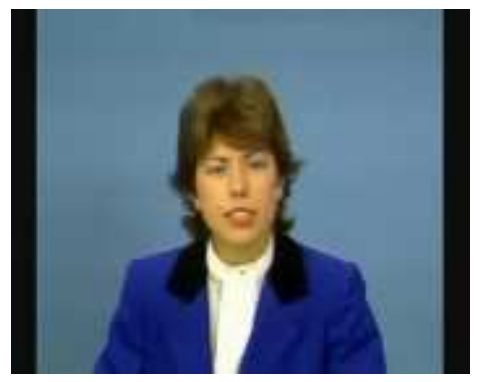

(c)

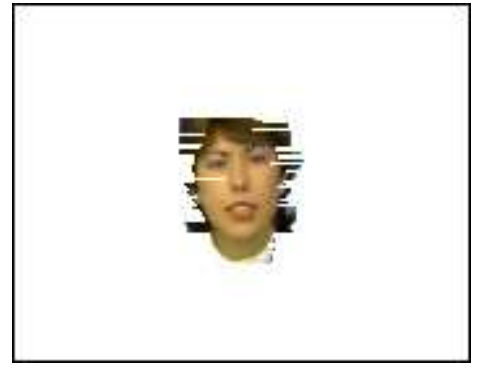

(b)

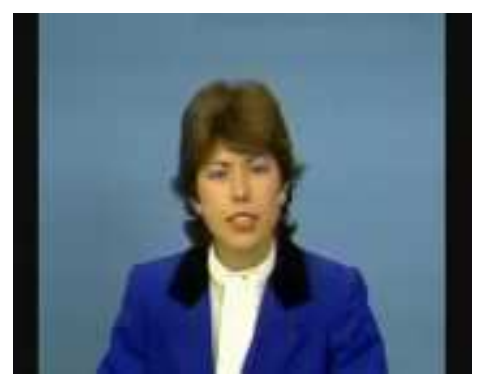

(d)

Figure 4.4: Comparison between virtual-object compression and Single Arbitrarily Shaped Virtual-Object Compression using "CLAIRE" sequence (frame \#22 is shown), (a)Extracted Rectangular Virtual-Object (the colored portion only) in rectangular virtual-object compression (b) Extracted Single Arbitrarily Shaped Virtual Object (colored portion in the frame) (c) Rectangular virtual-object compression. Compression Ratio $=53: 1$, Average PSNR $=26.16(\mathrm{~d})$ Single Arbitrarily Shaped Virtual-Object Compression. Compression Ratio $=60.02: 1$, Average PSNR $=37.12$

demonstrates the compression results comparison. Again, the GoF size was set to 16 frames.

Additional comparisons were made using "10TV" and "AKIYO" sequences. Single arbitrarily shaped virtual-object compression as well as multiple arbitrarily shaped virtual-objects compression outperforms rectangular virtual-object compression consistently as shown in Figure 4.6. 


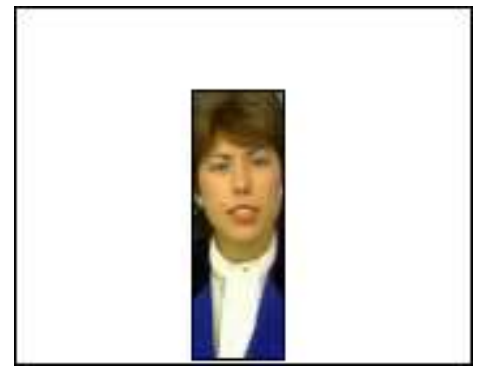

(a)

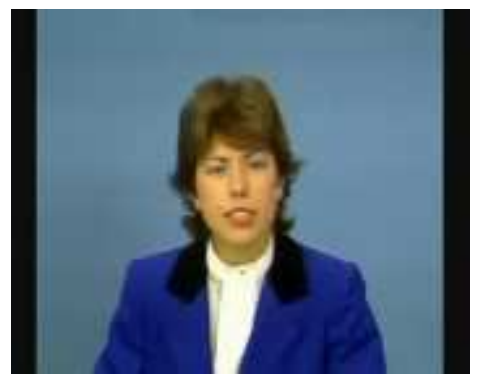

(c)

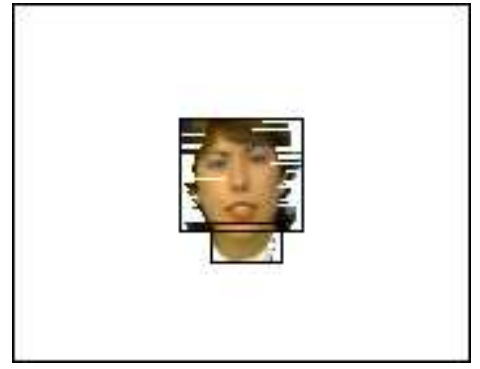

(b)

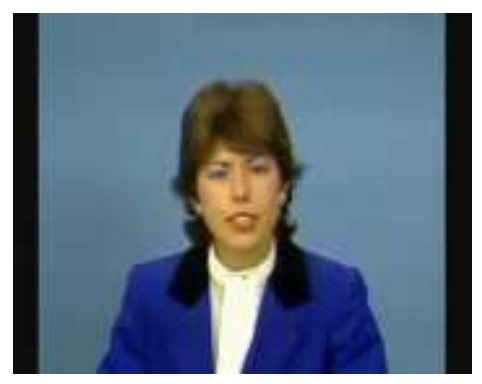

(d)

Figure 4.5: Comparison between virtual-object compression and Single Arbitrarily Shaped Virtual-Object Compression using "CLAIRE" sequence (frame \#22 is shown), (a)Extracted Rectangular Virtual-Object (the colored portion only)in rectangular virtual-object compression (b) Extracted Multiple Arbitrarily Shaped Virtual Objects (colored portion in the frame) (c) Rectangular virtual-object compression. Compression Ratio $=53: 1$, Average PSNR $=26.16$ (d)Multiple Arbitrarily Shaped Virtual-Objects Compression. Compression Ratio $=58.19: 1$, Average PSNR $=37.16$

Table 4.1 summarizes the results of comparison ratio (CR) and PSNR comparison between 3D wavelet, rectangular virtual-object (RVO), single arbitrarily shaped virtual-object (SASVO), and multiple arbitrarily shaped virtual-objects (MASVO) compression methods. 


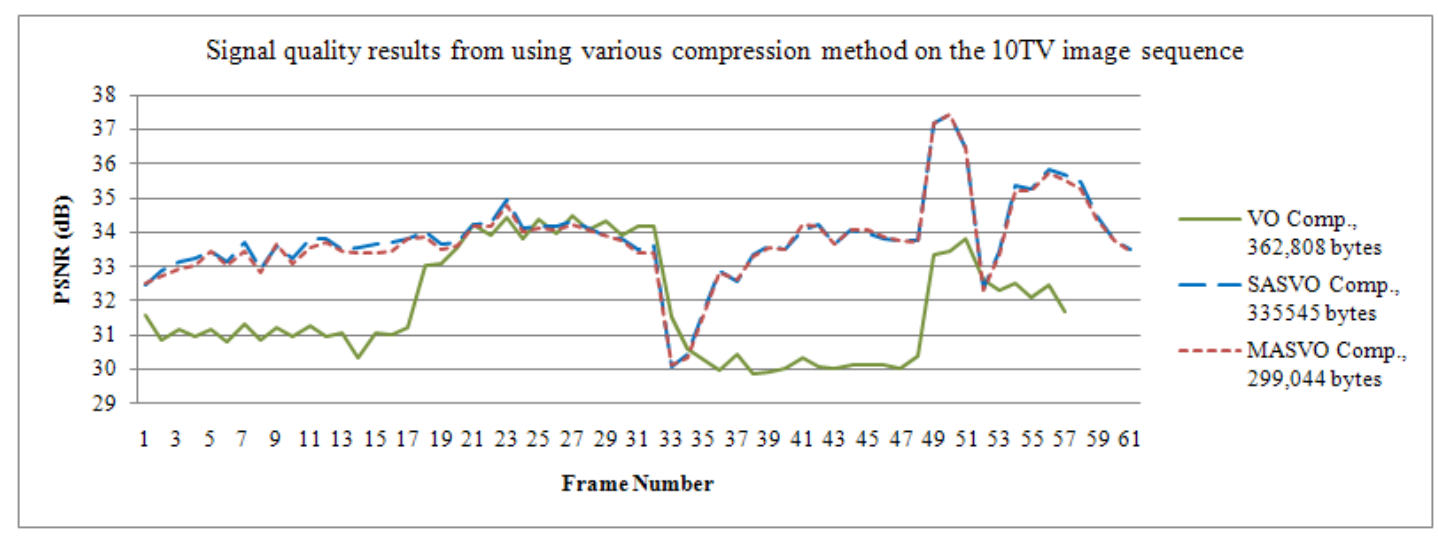

(a)

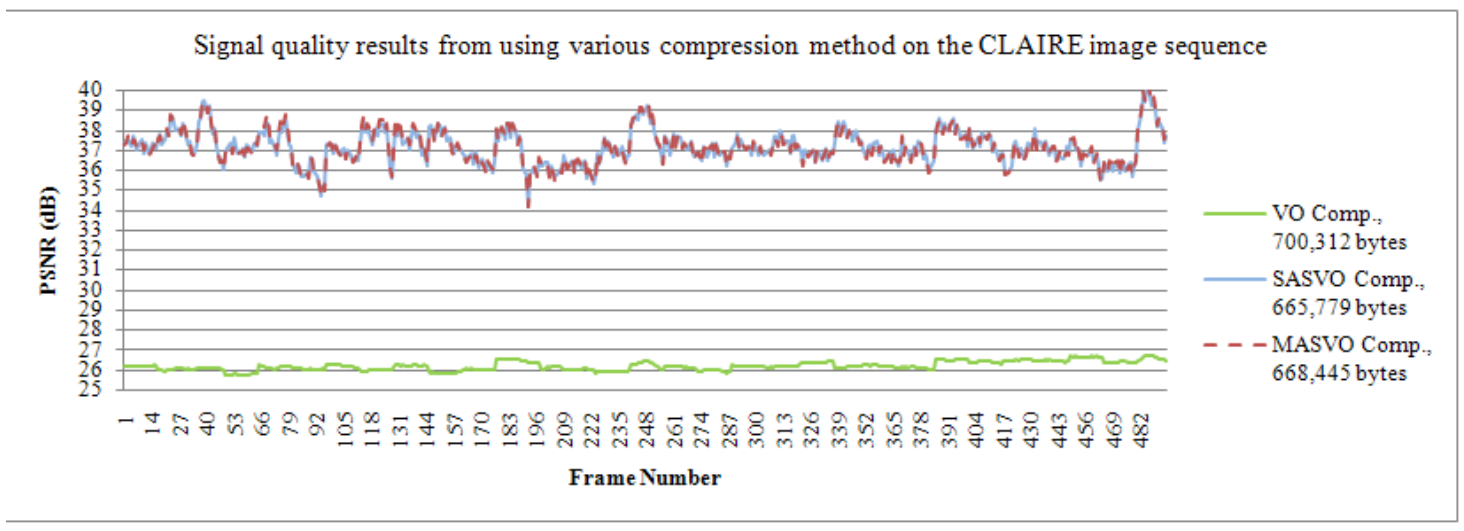

(b)

Signal quality results from using various compression method on the AKIYO image sequence

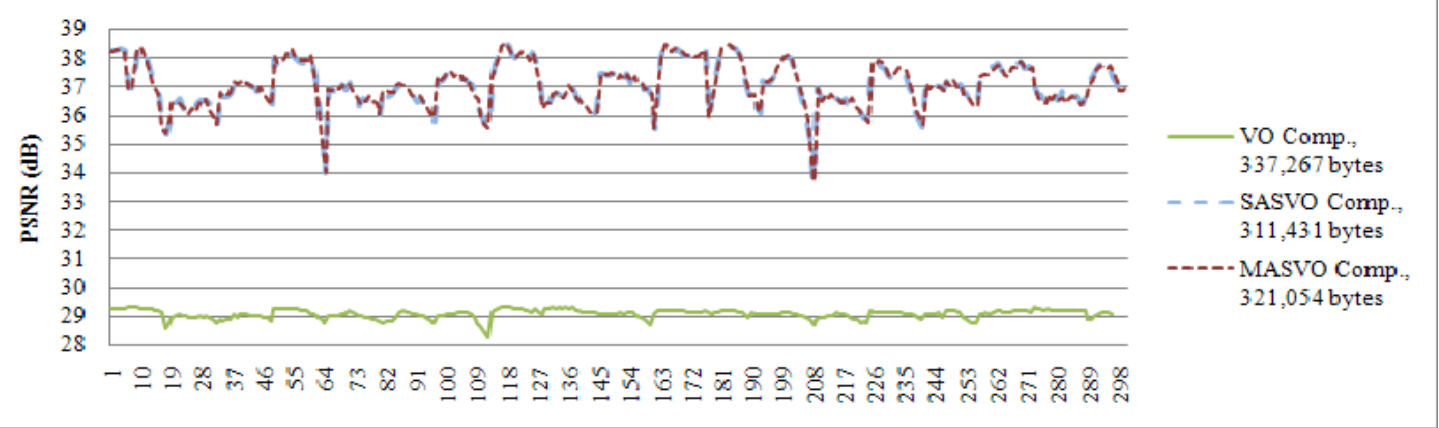

(c)

Figure 4.6: PSNR comparison between rectangular virtual-object (VO), single arbitrarily shaped virtual-object (SASVO), and multiple arbitrarily shaped virtual-object (MASVO) compression methods 


\begin{tabular}{|c|c|c|c|c|c|c|c|c|}
\hline \multirow[t]{2}{*}{$\begin{array}{l}\text { Images } \\
\text { Sequence }\end{array}$} & \multicolumn{2}{|c|}{$\begin{array}{l}\text { 3D Wavelet } \\
\text { Compression }\end{array}$} & \multicolumn{2}{|c|}{$\begin{array}{c}\text { RVO } \\
\text { Compression }\end{array}$} & \multicolumn{2}{|c|}{$\begin{array}{c}\text { SASVO } \\
\text { Compression }\end{array}$} & \multicolumn{2}{|c|}{$\begin{array}{c}\text { MASVO } \\
\text { Compression }\end{array}$} \\
\hline & $\mathrm{CR}$ & PSNR & $\mathrm{CR}$ & PSNR & $\mathrm{CR}$ & PSNR & $\mathrm{CR}$ & PSNR \\
\hline $10 \mathrm{TV}$ & $23.00: 1$ & 31.53 & $23.00: 1$ & 31.87 & $42.22: 1$ & 33.83 & $47.00: 1$ & 33.76 \\
\hline AKIYO & 46.00:1 & 29.44 & $67.00: 1$ & 29.10 & $73.24: 1$ & 37.06 & $72.05: 1$ & 37.08 \\
\hline CLAIRE & 43.00:1 & 26.23 & 53.00:1 & 26.16 & $60.02: 1$ & 37.12 & 58.19:1 & 37.16 \\
\hline
\end{tabular}

Table 4.1: Summary of Compression results

\subsubsection{Video Sequence with Multiple Moving Objects}

We have seen that arbitrarily shaped virtual-object compression outperforms rectangular virtual-object compression and 3D wavelet compression consistently for image sequence having just one moving object. Moreover, if we apply multiple arbitrarily shaped virtual-object compression to such video sequences, results are quite same as single arbitrarily shaped virtual-object compression as expected because there is only one moving object in the video scene. However, if there are multiple moving objects in the sequence, then multiple arbitrarily shaped virtual-object compression can perform better than the single arbitrarily shaped virtual-object compression. Therefore, it is interesting to test the behavior of these compression methods for video sequences involving multiple moving objects. One such sequence involving multiple objects is shown in Figure 4.7.

Rectangular virtual-object, single arbitrarily shaped virtual-object as well as multiple arbitrarily shaped virtual-object compression methods are applied to this image sequence. Figure 4.8 shows the virtual-objects extracted using various compression methods. Virtual-object portion is shown in real image colors whereas stationary background portion is shown in white for the purpose of clarity. 


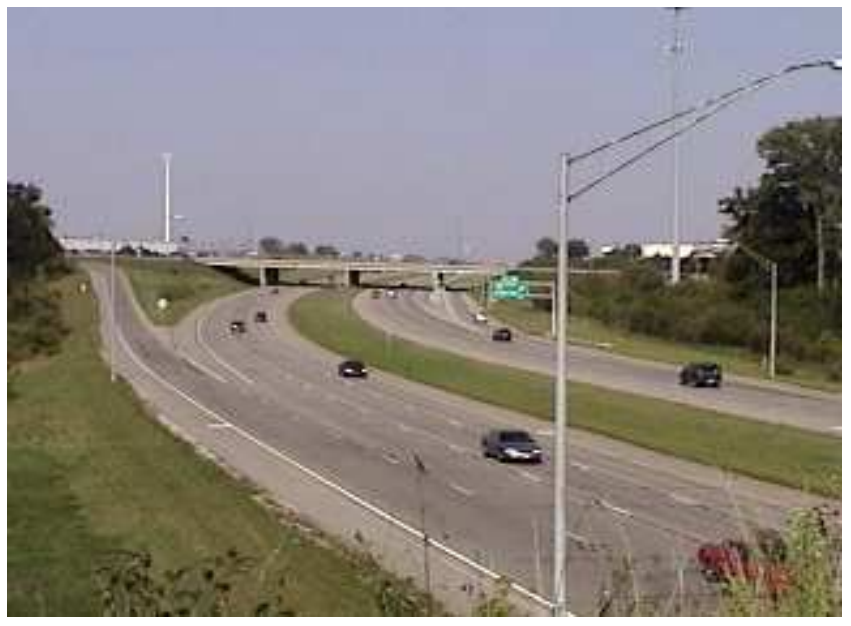

(a)

Figure 4.7: Image Sequence with multiple moving objects

Clearly from Figure 4.8, the area belonging to the virtual-object in the spatial domain is largest for rectangular virtual-object extraction. As a result compression ratio will be less for rectangular virtual-object compression method. Figure 4.9 shows the compression ratio and the corresponding average PSNR for the various virtual-object based compression methods.

Therefore, multiple arbitrarily shaped virtual-objects compression gives the best compression results in this case and with a higher PSNR value. Overall, arbitrarily shaped virtual-object compression is much superior to the rectangular virtual-object based compression.

\subsection{Conclusions}

Arbitrarily shaped virtual-object method has been shown to be performing much better than pure 3D wavelet compression as well as shown to be superior to the 


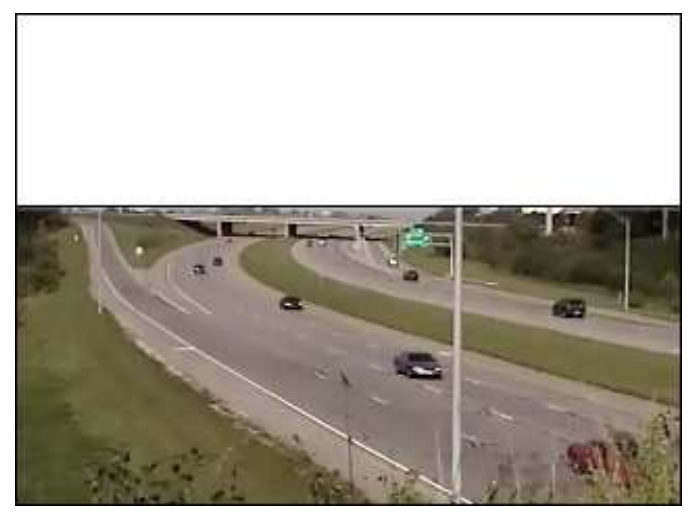

(a)

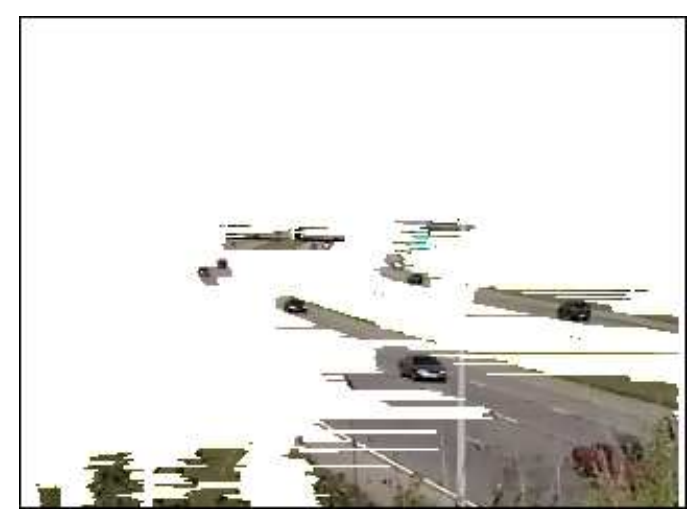

(b)

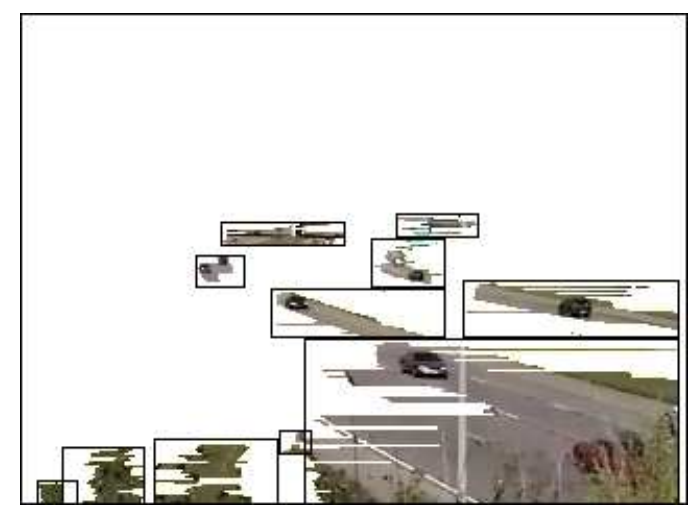

(c)

Figure 4.8: Virtual-object extraction. (a) Rectangular virtual-object (b) Single arbitrarily shaped virtual-object (c) Multiple arbitrarily shaped virtual-objects and their bounding boxes

rectangular virtual-object compression. The newly presented method consistently outperforms 3D wavelet compression and rectangular virtual-object compression by achieving higher compression ratios and higher PSNR values. In addition, multiple arbitrarily shaped virtual-object compression is shown to be working better than the single arbitrarily shaped virtual-object compression in case of video sequence with multiple objects. Therefore, multiple arbitrarily shaped virtual-objects compression 


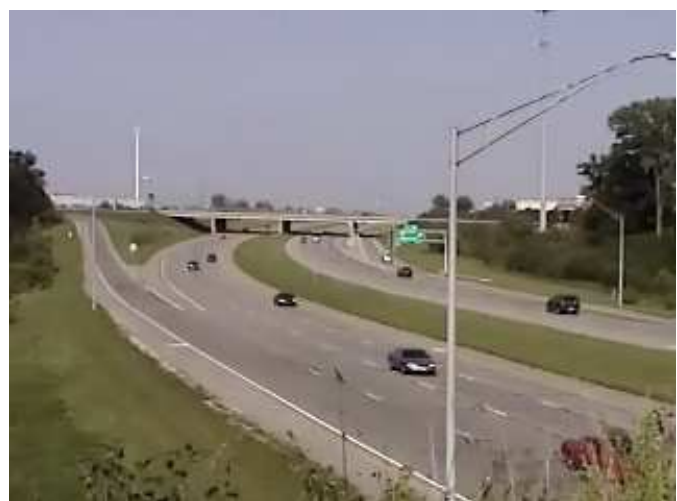

(a)

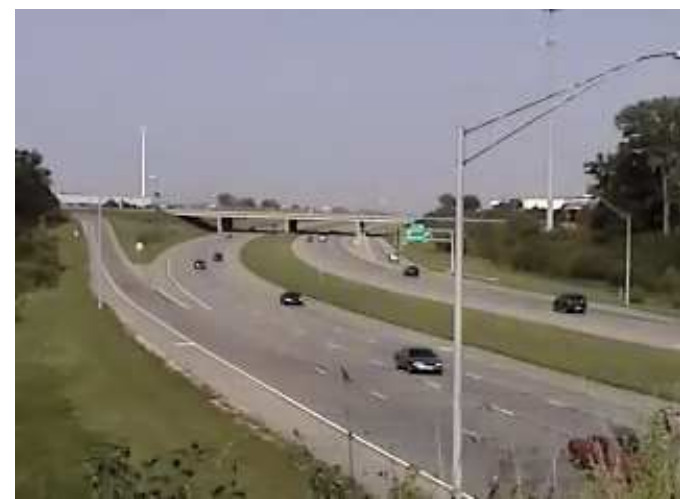

(b)

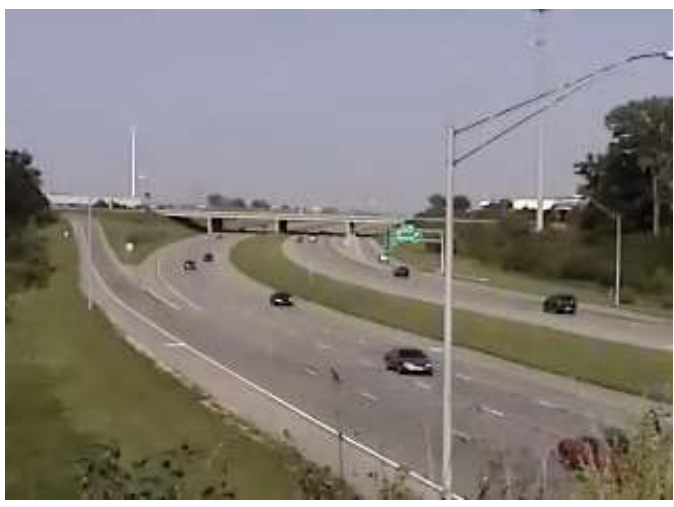

(c)

Figure 4.9: Performance Comparisons. (a) Rectangular virtual-object compression. Compression Ratio $=25: 1$, Average PSNR $=29.85$, (b) Single arbitrarily shaped virtual-object compression. Compression Ratio $=47.91: 1$, Average PSNR $=31.21$, and (c) Arbitrarily shaped multiple virtual-objects compression. Compression Ratio $=49.45: 1$, Average PSNR $=31.44$ 
can be used to further improve compression results in case of video sequence with multiple objects though it is subjected to experimental conditions as explained in subsection 3.3.3. Furthermore, the newly proposed method is compared to the latest compression standards and give quite comparable results at a very low computational cost. Overall, arbitrarily shaped virtual-object compression method gives better compression results than the rectangular virtual-object, and 3D wavelet compression and quite comparable results to the latest compression standards such as MPEG-4 ASP, and H264. 


\section{CHAPTER 5}

\section{CONTRIBUTIONS AND FUTURE WORK}

We have presented an arbitrarily shaped virtual-object video compression method that improves the compression ratio possible for a rectangular virtual-object method. At the same time, the presented method also provides some of the benefits of the object-based compression method like MPEG-4. This chapter summarizes the research presented and the contributions made. A few topics for the future research are also presented.

\subsection{Contributions}

Object based video compression has been an active area of research for quite some time now. However, true object extraction is computationally very expensive. Similarly, there is a significant interest in the usage of wavelet transform for video compression as wavelet transform based compression techniques have been shown to outperform discrete cosine transform based compression techniques in the field of image compression.

Therefore, a shape adaptive wavelet transform based arbitrarily shaped virtualobject video compression method is developed. The method further explores wavelet transform based video compression and also, provides some of the benefits of the 
object based compression for a really low computational cost. In arbitrarily shaped virtual-object compression, arbitrarily shaped virtual-object is separated from the stationary background. The arbitrarily shaped virtual object is compressed using 3D wavelet compression with only difference from the 3D wavelet compression being that the spatial decomposition is done using shape adaptive wavelet transform. The background is compressed using $2 \mathrm{D}$ wavelet compression. The method provides better compression ratio than the pure 3D wavelet compression as well as better compression ratio than the rectangular virtual-object compression for the same PSNR.

Furthermore, multiple arbitrarily shaped virtual-object compression method is presented. Encoding each arbitrarily shaped objects separately from others usually results in an increase in coding efficiency and therefore, should result in an increase in compression ratio. However, in the multiple arbitrarily shaped virtual-object compression method, we also need to encode the positions of the bounding boxes of the different arbitrarily shaped objects which can decrease the compression ratio. Therefore, compression ratio will increase or decrease subjected to experiment under consideration in case of multiple arbitrarily shaped virtual-object compression. In any case though, arbitrarily shaped virtual-object compression outperforms 3D wavelet compression and rectangular virtual-object compression.

\subsection{Future Works}

The method presented here uses a shape adaptive wavelet transform method to improve the compression ratio possible for the wavelet transform based virtual object video compression. Although the method achieves good results compared to some 
other object or wavelet based compression methods, there is still scope for further improvements. Below we list few areas which can be further explored.

- The most important step in object based compression method is to extract moving object accurately and in a computationally efficient manner. Today we have object extraction methods that can extract the object accurately but these are too computationally expensive to be implemented in a real-time video compression method. The method we developed as well as many other object based compression methods extract an approximation to the actual object where some background pixels are also extracted as part of the object and therefore, such methods can not achieve the true potential of object based compression. Therefore, a computationally efficient object extraction method needs to be developed which can optimize the object based compression potential.

- The wavelet function to be used for video compression is determined experimentally most of the time. However, different input signals respond differently to different wavelet transforms and therefore, same type of wavelet function can not be used for all types of input signals. Further research can be conducted to find out what types of wavelet functions are suitable for a given type of input signals.

- The shape adaptive wavelet transform used for spatial decomposition is a computationally expensive method as well as consumes more memory than the conventional wavelet transform. Though some computationally efficient methods are available for some types of wavelets, further research can be carried out 
to make shape adaptive wavelet transform method less memory consuming and more computationally efficient.

- The method works great for videos with stationary backgrounds. However, in case the background is changing or if the camera is in motion, new compression method will give the same performance as for 3D wavelet compression. Further research can be carried out to take the camera motion into consideration 


\section{BIBLIOGRAPHY}

[1] ISO/IEC 11172-2. Information Technology - Coding of Moving Pictures and Associated Audio for Digital Storage Media at up to about $1.5 \mathrm{Mbits} / \mathrm{s}$ Part 2: Video, Mar 1993.

[2] ISO/IEC 13818-2. Information Technology - Generic Coding of Moving Pictures and Associated Audio Information : Video, Mar 1995.

[3] ISO/IEC 14496-10. Information technology - Coding of Audio-Visual Objects : MPEG-4 Systems, Oct 1998.

[4] ISO/IEC 14496-10. Information technology - Coding of Audio-Visual ObjectsPart 10 : Advanced Video Coding, Oct 2004.

[5] A. Abu-Hajar and R. Sankar. Integer-to-Integer Shape Adaptive Wavelet Transform for Region of Interest Image Coding. In Proc. IEEE 10th Digital Signal Processing Workshop, and the 2nd Signal Processing Education Workshop, pages 94-97, Oct 2002.

[6] R. Adhami. Video Compression Technique Using Wavelet Transform. In Proc. IEEE Conf. Aerospace Applications, 1996.

[7] E. J. Balster and Y. F. Zheng. Virtual-Object Video Compression. In Proc. 48th Midwest Symposium on Circuits and Systems, pages 1700-1704, Aug 2005.

[8] E. J. Balster, Y. F. Zheng, and R. L. Ewing. Feature-Based Wavelet Shrinkage Algorithm for Image Denoising. IEEE Trans. Image Processing, 14(12):20242039, Dec 2005.

[9] E. J. Balster, Y. F. Zheng, and R. L. Ewing. Combined Spatial and Temporal Domain Wavelet Shrinkage Algorithm for Video Denoising. IEEE Trans. Circuits and Systems for Video Technology, 16(2):220-230, Feb 2006.

[10] T. S. Bindulal and M. R. Kaima. Object Coding Using a Shape Adaptive Wavelet Transform with Scalable WDR Method. In Proc. IEEE Intl. Conf. Image Processing, volume 2, pages 325-328, Oct 2007. 
[11] Berkeley Multimedia Research Center. MPEG-1 faq, Aug 2001.

[12] Berkeley Multimedia Research Center. MPEG-2 faq, Aug 2001.

[13] C. Christopoulos, A. Skodras, and T. Ebrahimi. The JPEG2000 Still Image Coding System: An Overview. IEEE Trans. Consumer Electronics, 46(4):11031127, Nov 2000.

[14] C. He, J. Dong, Y. F. Zheng, and Z. Gao. Optimal 3-D Coefficient Tree Structure for 3-D Wavelet Video Coding. IEEE Trans. Circuits and Systems for Video Technology, 13(10):961-972, Oct 2003.

[15] C. He, Y. F. Zheng, and S. C. Ahalt. Object Tracking Using the Gabor Wavelet Transform and the Golden Section Algorithm. IEEE Trans. Multimedia, 4(4):528-538, Dec 2002.

[16] Y. T. Hwang, Y. C. Wang, and S. S. Wang. An Efficient Shape Coding Scheme and its Codec Design. pages 225-232, 2001.

[17] J. Li and S. Lei. Arbitrary Shape Wavelet Transform with Phase Alignment. In Proc. IEEE Intl. Conf. Image Processing, volume 3, pages 683-687, Oct 1998.

[18] S. Li and W. Li. Shape-Adaptive Discrete Wavelet Transforms for Arbitrarily Shaped Visual Object Coding. IEEE Trans. Circuits and Systems for Video Technology, 10(5):725-743, Aug 2000.

[19] C. Lin, B. Zhang, and Y. F. Zheng. Packed Integer Wavelet Transform constructed by Lifting Scheme. IEEE Trans. Circuits and Systems for Video Technology, 10(8):1496-1501, Dec 2000.

[20] Y. Liu, K. N. Ngan, and F. Wu. 3-D Shape-Adaptive Directional Wavelet Transform for Object-Based Scalable Video Coding. IEEE Trans. Circuits and Systems for Video Technology, 18(7):888-899, Jul 2008.

[21] S. A. Martucci, I. Sodagar, T. Chiang, and Y. Zhang. A Zerotree Wavelet Video Coder. IEEE Trans. Circuits and Systems for Video Technology, 7(1):109-118, Feb 1997.

[22] U. Y. Oktiawati and V. V. Yap. Video Compression Using Dual Tree Complex Wavelet Transform. In Proc. Intl. Conf. Intelligent and Advanced Systems, pages 775-778, Nov 2007.

[23] A. Polzer, H. Klock, and J. M. Buhrmann. Video Coding by Region-Based Motion Compensation and Spatio-Temporal Wavelet Transform. In Proc. Intl. Conf. Image Processing, volume 3, pages 436-439, Oct 1997. 
[24] A. Said and W. A. Pearlman. A New, Fast, and Efficient Image Codec based on Set Partitioning in Hierarchical Trees. IEEE Trans. Circuits and Systems for Video Technology, 6(3):243-250, Jun 1996.

[25] P. Seigneurbieux and Z. Xiong. Progressive Trellis-Coded Space-Frequency Quantization for Wavelet Image Coding. IEEE Trans. Circuits and Systems for Video Technology, 12(7):587-591, Jul 2002.

[26] Z. Shen, M. R. Frater, and J. F. Arnold. Quad-Tree Block-Based Binary Shape Coding. IEEE Trans. Circuits and Systems for Video Technology, 18(6):845-850, Jun 2008.

[27] T. Sikora and B. Makai. Shape-Adaptive DCTfor Generic Coding of Video. IEEE Trans. Circuits and Systems for Video Technology, 5(1):59-62, Feb 1995.

[28] T. M. Strat. Object-Based Encoding: Next-Generation Video Compression. In Proc. Workshop and Exhibition. MPEG-4, pages 53-57, Jun 2001.

[29] W. Sweldens. The Lifting Scheme: A Custom Design Construction of Biorthogonal Wavelets. Appl. Comput.Harmon. Anal, 3(2):186-200, Apr 1996.

[30] W. Tan, E. Chan, and A. Zalchor. Real Time Software Implementation of Scalable Video Codec. In Proc. Intli. Conf. Image Processing, volume 1, pages 17-20, Sep 1996.

[31] J. Y. Tham, S. Ranganath, and A. A. Kassim. Highly Scalable Wavelet-Based Video Codec for Very Low Bit-Rate Environment. IEEE Trans. Selected Areas in Communications, 16(1):12-27, Jan 1998.

[32] M. J. Tsai, J. D. Villasenor, and F. Chen. Stack-Run Image Coding. IEEE Trans. Circuits and Systems for Video Technology, 6(5):519-521, Oct 1996.

[33] P. Viola and M. Jones. Rapid Object Detection Using a Boosted Cascade of Simple Features. In Proc. IEEE Computer Society Conf. Computer Vision and Pattern Recognition, volume 1, pages 511-518, 2001.

[34] G. Xing, J. Li, S. Li, and Y. Q. Zhang. Arbitrarily Shaped Video-Object Coding by Wavelet. IEEE Trans. Circuits and Systems for Video Technology, 11(10):1135-1139, Oct 2001.

[35] Z. Xiong, K. Ramchandran, M. T. Orchard, and Y. Zhang. A Comparative Study of DCT and Wavelet Based Coding. In Proc. IEEE Intl. Symp. Circuits and Systems, volume 4, pages 273-276, Jun 1998.

[36] L. Zhou and S. Zahir. A Novel Shape Coding Scheme For MPEG-4 Visual Standard. 3:585-588, Sep 2006. 